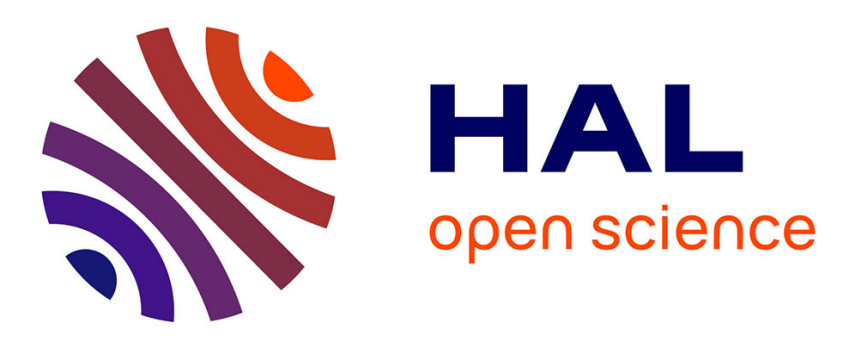

\title{
Fully decoupled time-marching schemes for incompressible fluid/thin-walled structure interaction
}

Miguel Angel Fernández, Mikel Landajuela, Marina Vidrascu

\section{To cite this version:}

Miguel Angel Fernández, Mikel Landajuela, Marina Vidrascu. Fully decoupled time-marching schemes for incompressible fluid/thin-walled structure interaction. Journal of Computational Physics, 2015, 297, pp.156-181. 10.1016/j.jcp.2015.05.009 . hal-00918498v4

\section{HAL Id: hal-00918498 \\ https://inria.hal.science/hal-00918498v4}

Submitted on 6 May 2015

HAL is a multi-disciplinary open access archive for the deposit and dissemination of scientific research documents, whether they are published or not. The documents may come from teaching and research institutions in France or abroad, or from public or private research centers.
L'archive ouverte pluridisciplinaire HAL, est destinée au dépôt et à la diffusion de documents scientifiques de niveau recherche, publiés ou non, émanant des établissements d'enseignement et de recherche français ou étrangers, des laboratoires publics ou privés. 
Fully decoupled time-marching schemes for incompressible fluid/thin-walled structure interaction

Miguel A. Fernández, Mikel Landajuela, Marina Vidrascu

\section{RESEARCH}

REPORT

$\mathrm{N}^{\circ} 8425$

December 2014

Project-Team Reo 



\title{
ching
}

\section{Fully decoupled time-marching schemes for incompressible fluid/thin-walled structure interaction}

\author{
Miguel A. Fernández ${ }^{* \dagger}$, Mikel Landajuela ${ }^{* \dagger}$, Marina Vidrascu* ${ }^{* \dagger}$ \\ Project-Team Reo \\ Research Report n 8425 - December 2014 - 38 pages
}

\begin{abstract}
In this paper we introduce a class of fully decoupled time-marching schemes (velocitypressure-displacement splitting) for the coupling of an incompressible fluid with a thin-walled elastic or viscoelastic structure. The time splitting combines a projection method in the fluid with a specific Robin-Neumann treatment of the interface coupling. A priori energy estimates guaranteeing unconditional stability are established for some of the schemes. The accuracy and performance of the methods proposed is illustrated by a thorough numerical study.
\end{abstract}

Key-words: fluid-structure interaction, incompressible fluid, Reissner-Mindlin shell, timediscretization, projection method, explicit coupling scheme.

This work was supported by the French National Research Agency (ANR) through the EXIFSI project (ANR-12-JS01-0004)

* Inria, REO project-team, Rocquencourt - B.P. 105, F-78153 Le Chesnay cedex, France

† UPMC UniversitÃl' Paris VI, REO project-team, UMR 7958 LJLL, F-75005 Paris, France

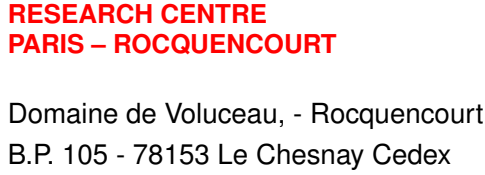




\section{Schémas de couplage totalement découplés pour l'interaction d'un fluide incompressible et une structure mince}

Résumé : Dans cet article, nous introduisons une classe de schémas entièrement découplés (vitesse-pression-déplacement) pour le couplage d'un fluide incompressible avec une structure Ál'lastique ou viscoélastique mince. Les méthodes combinent une discrétisation à pas fractionnaire globale du système couplé avec un traitement spécifique Robin-Neumann du couplage à l'interface. Des estimations d'énergie a priori garantissent la stabilité inconditionnelle des variantes sans extrapolation et avec extrapolation d'ordre un. La précision et la robustesse des méthodes proposées sont illustrées dans plusieurs exemples numériques.

Mots-clés : interaction fluide-structure, fluide incompressible, modèle de coque ReissnerMindlin, discrétisation en temps, méthode de projection, schéma de couplage explicit. 


\section{Introduction}

Mathematical problems describing the mechanical interaction of an elastic thin-walled structure with an incompressible fluid flow appear in a wide variety of engineering fields: from the aeroelasticity of sailing boats and parachutes, to sloshing dynamics in tanks, heat exchangers design, micro-encapsulation technology and the biomechanics of animal cells and physiological flows (see, e.g., [52, 69, 28, 61, 63, 71, 44, 55]).

Typically, these coupled problems (and other multi-physics problems in general) are discretized by exploiting the heterogeneous nature of the mechanical system. For instance, the fluid and the solid equations are often discretized by different time-stepping schemes adapted to their distinct mathematical properties. The time-discretization of the coupling conditions determines the so-called coupling scheme: implicit, semi-implicit and explicit (see, e.g., [29, 46, 25] for recent reviews).

With an implicit coupling scheme no time lag exits between the fluid and solid time-marchings. This can deliver unconditional stability and optimal accuracy, but at the price of solving a computationally demanding coupled problem at each time-step. The corresponding solution procedures are traditionally referred to in the literature as: monolithic and partitioned. Monolithic methods solve the coupled problem as a single system of equations (see, e.g., [5, 66, 39, 24, 56]). Partitioned methods, on the contrary, exploit the heterogenous nature of the system via (recurrent) separate solutions of the fluid and solid equations, with appropriate interface conditions (see, e.g., [34, 4, 26, 8, 58]). Partitioned solution procedures are very appealing because of their intrinsic modularity, which enables the reuse of independent efficient solvers. Such an advantage comes however at a price, computational efficiency over a monolithic approach is not necessarily guaranteed (see, e.g., [5, 39]).

Stable and less computationally onerous alternatives to implicit coupling are the so-called semi-implicit coupling schemes. These methods enforce a specific explicit/implicit treatment of the interface coupling conditions (see, e.g., [57, 51]) and are often combined with a fractional-step time-marching in the fluid (see, e.g., $[32,65,6,2,3]$ ) or in the solid (see, e.g., [42, 30, 17, 53]). The implicit part of the coupling (which, as above, can be solved in a monolithic or a partitioned fashion) guarantees stability, while the explicit one reduces computational complexity.

Explicit coupling schemes (also termed loosely coupled) uncouple the fluid and solid timemarchings via appropriate explicit discretizations of the interface conditions. The resulting solution procedures are thus naturally partitioned. The design and the analysis of stable and accurate explicit coupling schemes for incompressible fluid-structure interaction problem is a challenging problem. This is due to the fact that the interface coupling can be extremely stiff (see, e.g., [20]). Though stability has been an open problem for years (see [18]), the most intricate issue appears to be accuracy. The explicit coupling schemes reported in [18, 19] guarantee stability but at the expense of a degradation of accuracy, which requires suitable correction iterations. In the case of the coupling with an elastic thin-walled solid, unconditional stability is achieved with the explicit coupling scheme introduced in [42], which is known to yield very poor accuracy (see [30,31]). Numerical evidence suggests that enhanced accuracy can be obtained with the variants recently reported in $[17,53]$. Unfortunately, if physical damping is present in the structure equations, these coupling schemes are no longer explicit. These issues are overcome by the Robin-Neumann based explicit coupling schemes proposed in [36], which simultaneously deliver unconditional stability and optimal (first-order) time accuracy. A fundamental ingredient in the stability and accuracy of these methods is a specific combination of the interface Robin consistency of the coupled problem with a monolithic time-stepping in the fluid.

Since the pioneering work by Chorin and Temam (see [23, 70]), projection methods have become one of the most widespread techniques for the numerical solution of the incompressible 
Navier-Stokes equations in primitive variables (see, e.g., [64, 16, 41, 7] and the references therein). These methods segregate the computation of the velocity and of the pressure in terms of two decoupled elliptic problems which make them very appealing for large scale computations. The contribution of the present paper is to show how the explicit Robin-Neumann paradigm of [36] can be effectively used with a projection based time-marching in the fluid. An approach in this direction, intended to deliver second-order accuracy, has been recently reported in [12]. It is however not clear how to implement the interface splitting therein within a finite element framework.

The key idea of the schemes proposed in this paper lies in the derivation of an intrinsic fractional-step time-stepping of the interface Robin consistency. This preserves the stability and accuracy of the original Robin-Neumann splitting without compromising the velocity/pressure uncoupling in the fluid time-marching. In particular, the resulting solution procedures enable a fully decoupled computation of the whole fluid-solid state. The velocity/pressure splitting in the fluid introduces additional perturbations of the kinematic coupling which make the analysis much more intricate than in [36]. For a linear coupled problem involving the Stokes equations and a general (Reissner-Mindlin type) viscoelastic shell model, a priori energy estimates guaranteeing unconditional stability are derived for some of the variants. The proposed fully decoupled schemes are also formulated within a non-linear framework, involving the incompressible Navier-Stokes equations (in moving domains) and a non-linear viscoelastic shell model. A thorough numerical study, based on different linear and non-linear fluid-structure interaction examples, illustrates the accuracy and performance of the methods proposed.

The rest of the paper is organized as follows. Section 2 is devoted to the derivation and the analysis of the methods within a linear representative setting. In Section 3, the proposed fully decoupled schemes are formulated within a non-linear setting. The numerical results are presented and discussed in Section 4. Finally, a summary of the conclusions and some directions of further investigation are given in Section 5.

Some preliminary results of the present work have been announced, without proof, in [33].

\section{Derivation and analysis in the linear case}

We consider a simplified linear model problem in which the fluid is described by the Stokes equations, in a fixed domain, and the structure by a linear viscoelastic Reissner-Mindlin shell model (see, e.g., [21, 13]). Basically, we assume that the displacements of the shell are infinitesimal and that the Reynolds number in the fluid is small. We denote by $\Omega \subset \mathbb{R}^{3}$ the fluid domain and by $\partial \Omega$ its boundary. The fluid boundary is partitioned as $\partial \Omega=\Gamma \cup \Sigma$, where $\Sigma$ stands for the fluid-structure interface. Since the structure is thin-walled, the interface $\Sigma$ is itself the reference configuration of the shell mid-surface (see Remark 2 below). The exterior unit-vector normal to $\partial \Omega$ is denoted by $\boldsymbol{n}$. For a given vector field $\boldsymbol{v}$ defined on the surface $\Sigma$, the symbols $\boldsymbol{v}_{\perp} \stackrel{\text { def }}{=}(\boldsymbol{v} \cdot \boldsymbol{n}) \boldsymbol{n}$ and $\boldsymbol{v}_{\|} \stackrel{\text { def }}{=} \boldsymbol{v}-\boldsymbol{v}_{\perp}$ will denote, respectively, the normal and tangential components of $\boldsymbol{v}$.

The resulting coupled problem reads as follows: find the fluid velocity $\boldsymbol{u}: \Omega \times \mathbb{R}^{+} \rightarrow \mathbb{R}^{3}$, the pressure $p: \Omega \times \mathbb{R}^{+} \rightarrow \mathbb{R}$, the solid displacement $\boldsymbol{d}: \Sigma \times \mathbb{R}^{+} \rightarrow \mathbb{R}^{3}$ and the rotation vector $\boldsymbol{\theta}: \Sigma \times \mathbb{R}^{+} \rightarrow \mathbb{R}^{3}$, satisfying the Reissner-Mindlin kinematical assumption $\boldsymbol{\theta}_{\perp}=\mathbf{0}$, such that

$$
\left\{\begin{aligned}
\rho^{\mathrm{f}} \partial_{t} \boldsymbol{u}-\operatorname{div} \boldsymbol{\sigma}(\boldsymbol{u}, p)=\mathbf{0} & \text { in } \quad \Omega, \\
\operatorname{div} \boldsymbol{u}=0 & \text { in } \quad \Omega, \\
\boldsymbol{\sigma}(\boldsymbol{u}, p) \boldsymbol{n}=-p_{\Gamma} \boldsymbol{n} & \text { on } \quad \Gamma,
\end{aligned}\right.
$$




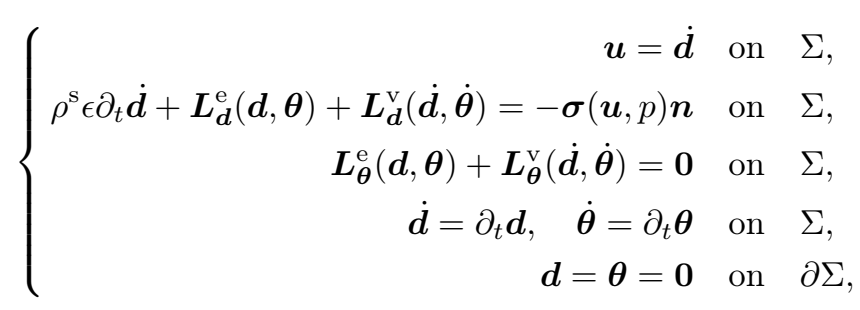

satisfying the initial conditions $\boldsymbol{u}(0)=\boldsymbol{u}_{0}, \boldsymbol{d}(0)=\boldsymbol{d}_{0}, \boldsymbol{\theta}(0)=\boldsymbol{\theta}_{0}$ and $\dot{\boldsymbol{d}}(0)=\dot{\boldsymbol{d}}_{0}$. The constants $\rho^{\mathrm{f}}$ and $\rho^{\mathrm{s}}$ denote, respectively, the fluid and solid densities and $\epsilon$ is the solid thickness. The fluid is assumed to be incompressible and Newtonian so that its Cauchy-stress tensor is given by the relation $\boldsymbol{\sigma}(\boldsymbol{u}, p) \stackrel{\text { def }}{=}-p \boldsymbol{I}+2 \mu \boldsymbol{\varepsilon}(\boldsymbol{u})$, with $\boldsymbol{\varepsilon}(\boldsymbol{u}) \stackrel{\text { def }}{=} \frac{1}{2}\left(\boldsymbol{\nabla} \boldsymbol{u}+\boldsymbol{\nabla} \boldsymbol{u}^{\mathrm{T}}\right)$ and where $\mu$ stands for the fluid dynamic viscosity. A given pressure data $p_{\Gamma}$ is prescribed on the fluid external boundary $\Gamma$. In this section, the elastic and viscous solid operators, $\left(\boldsymbol{L}_{\boldsymbol{d}}^{\mathrm{e}}, \boldsymbol{L}_{\boldsymbol{\theta}}^{\mathrm{e}}\right)$ and $\left(\boldsymbol{L}_{\boldsymbol{d}}^{\mathrm{v}}, \boldsymbol{L}_{\boldsymbol{\theta}}^{\mathrm{v}}\right)$, are supposed to be linear.

Remark 1 Problem (1)-(2) retains all the added-mass effect numerical issues that appear in complex non-linear incompressible fluid-structure interaction problems. The external boundary conditions of the coupled problem have no impact on the coupling schemes and theoretical results presented below.

Remark 2 As usual in shell dynamic analysis, the rotational inertial term is neglected in $(2)_{2}$. The interface conditions $(2)_{1,2}$ are enforced on the shell mid-surface $\Sigma$, instead of on the true fluid-solid interface. In other words, the shell thickness is neglected in the interface coupling. This is a widely used modeling simplification when coupling thin-walled solids with thick-walled materials (see, e.g., [22]).

\subsection{Time semi-discretization: fully decoupled schemes}

In the following text, the symbol $\tau$ denotes the time-step length, $t_{n} \stackrel{\text { def }}{=} n \tau$, for $n \in \mathbb{N}$, and $\partial_{\tau} x^{n} \stackrel{\text { def }}{=}\left(x^{n}-x^{n-1}\right) / \tau$ stands for the first order backward difference in time. We will also make extensive use of the superscripts ${ }^{\bullet}$ and ${ }^{\star}$ to respectively indicate explicit extrapolations of order $s \in\{0,1\}$ and $r \in\{0,1,2\}$, namely,

$$
x^{n, \bullet} \stackrel{\text { def }}{=}\left\{\begin{array}{lll}
0 & \text { if } \quad s=0, \\
x^{n-1} & \text { if } \quad s=1,
\end{array}, \quad x^{n, \star} \stackrel{\text { def }}{=}\left\{\begin{array}{lll}
0 & \text { if } & r=0, \\
x^{n-1} & \text { if } & r=1, \\
2 x^{n-1}-x^{n-2} & \text { if } & r=2 .
\end{array}\right.\right.
$$

The use of these two different notations will be made clear below (see Remark 3).

This section is devoted to the time discretization of the coupled problem (1)-(2). A well-known salient feature of this type of coupled systems is that it enforces an intrinsic Robin consistency on the interface (see, e.g., $[59,42,31,36])$. More specifically, from $(2)_{1,2}$ it follows that

$$
\boldsymbol{\sigma}(\boldsymbol{u}, p) \boldsymbol{n}+\rho^{\mathrm{s}} \epsilon \partial_{t} \boldsymbol{u}=-\boldsymbol{L}_{\boldsymbol{d}}^{\mathrm{e}}(\boldsymbol{d}, \boldsymbol{\theta})-\boldsymbol{L}_{\boldsymbol{d}}^{\mathrm{v}}(\dot{\boldsymbol{d}}, \dot{\boldsymbol{\theta}}) \quad \text { on } \quad \Sigma,
$$

which can be viewed as a Robin-like boundary condition for the fluid. This notion of interface Robin consistency has recently been used in the literature to avoid, without compromising optimal accuracy, the infamous unconditional instability issues of standard Dirichlet-Neumann loosely coupled schemes. Basically, these methods split the time-marching of $(\boldsymbol{u}, p)$ and $(\boldsymbol{d}, \boldsymbol{\theta})$ by combining a monolithic time-stepping of (1) with a specific time discretization of (3) (see, e.g., 
[36]). Our contribution in the present paper is to show how this explicit coupling paradigm can be effectively used with a projection method in the fluid. The resulting methods enable a fully decoupled sequential computation of the whole fluid-solid state: $\boldsymbol{u}, p$ and $(\boldsymbol{d}, \boldsymbol{\theta})$.

The solution procedures proposed in this paper build on the following three fundamental ingredients:

1. Projection based time-marching of the fluid:

(a) Viscous-step:

$$
\left\{\begin{array}{r}
\rho^{\mathrm{f}} \frac{\tilde{\boldsymbol{u}}^{n}-\boldsymbol{u}^{n-1}}{\tau}-\operatorname{div} \boldsymbol{\sigma}\left(\tilde{\boldsymbol{u}}^{n}, p^{n, \bullet}\right)=\mathbf{0} \quad \text { in } \quad \Omega, \\
\boldsymbol{\sigma}\left(\tilde{\boldsymbol{u}}^{n}, p^{n, \bullet}\right) \boldsymbol{n}=-p_{\Gamma}^{n, \bullet} \boldsymbol{n} \text { on } \quad \Gamma .
\end{array}\right.
$$

(b) Projection-step:

$$
\left\{\begin{aligned}
\rho^{\mathrm{f}} \frac{\boldsymbol{u}^{n}-\tilde{\boldsymbol{u}}^{n}}{\tau}+\nabla \phi^{n}=\mathbf{0} & \text { in } \Omega, \\
\operatorname{div} \boldsymbol{u}^{n}=0 & \text { in } \Omega, \\
\phi^{n}=p_{\Gamma}^{n}-p_{\Gamma}^{n, \bullet} & \text { on } \Gamma,
\end{aligned}\right.
$$

with $\phi^{n} \stackrel{\text { def }}{=} p^{n}-p^{n, \bullet}$.

We recall that the choices $s=0,1$ correspond, respectively, to the so-called non-incremental and incremental pressure-correction schemes (see, e.g, [41, Section 3]). The arguments below can be extended with ease to a velocity-correction time-stepping (see, e.g, [41, Section 4]) in the fluid.

2. Explicit interface conditions for (4) and (5), based on a specific fractional-step timemarching of (3), which preserve the velocity/pressure splitting and treat implicitly only the solid inertia contribution.

3. Fluid stresses are transmitted to the solid by solving the standard shell problem:

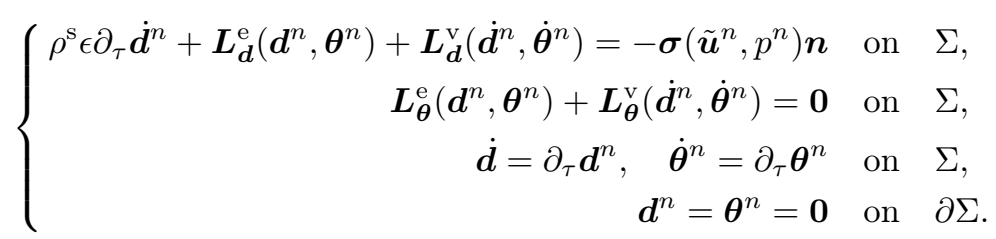

We now further elaborate on the second point. To this purpose, we first note that the projection-step (5) only contributes to the normal component of the fluid-stress, that is, $\boldsymbol{n}$. $\boldsymbol{\sigma}(\boldsymbol{u}, p) \boldsymbol{n}$. This is consistent with the fact that, in (5), only the normal component of the velocity has a well-defined trace on $\Sigma$. In terms of interface coupling, this indicates that the projectionstep only contributes to the normal component of (3). Therefore, its tangential component has to be taken into account in the viscous-step (4). This motivates the following two-stage time discretization of $(3)$ :

$$
\left\{\begin{array}{l}
\boldsymbol{\sigma}\left(\tilde{\boldsymbol{u}}^{n}, p^{n, \bullet}\right) \boldsymbol{n}+\frac{\rho^{\mathrm{s}} \epsilon}{\tau} \tilde{\boldsymbol{u}}^{n}=\frac{\rho^{\mathrm{S}} \epsilon}{\tau} \dot{\boldsymbol{d}}^{n-1}-\boldsymbol{L}_{\boldsymbol{d}}^{\mathrm{e}}\left(\boldsymbol{d}^{n, \star}, \boldsymbol{\theta}^{n, \star}\right)_{\|}-\boldsymbol{L}_{\boldsymbol{d}}^{\mathrm{v}}\left(\dot{\boldsymbol{d}}^{n, \star}, \dot{\boldsymbol{\theta}}^{n, \star}\right)_{\|}, \\
-\phi^{n}+\frac{\rho^{\mathrm{s} \epsilon}}{\tau} \boldsymbol{u}^{n} \cdot \boldsymbol{n}=\frac{\rho^{\mathrm{s}} \epsilon}{\tau} \tilde{\boldsymbol{u}}^{n} \cdot \boldsymbol{n}-\boldsymbol{L}_{\boldsymbol{d}}^{\mathrm{e}}\left(\boldsymbol{d}^{n, \star}, \boldsymbol{\theta}^{n, \star}\right) \cdot \boldsymbol{n}-\boldsymbol{L}_{\boldsymbol{d}}^{\mathrm{v}}\left(\dot{\boldsymbol{d}}^{n, \star}, \dot{\boldsymbol{\theta}}^{n, \star}\right) \cdot \boldsymbol{n}
\end{array}\right.
$$


Algorithm 1 Fully decoupled schemes for (1)-(2).

For $n>s+r$ :

1. Fluid viscous sub-step: find $\tilde{\boldsymbol{u}}^{n}: \Omega \rightarrow \mathbb{R}^{d}$ such that

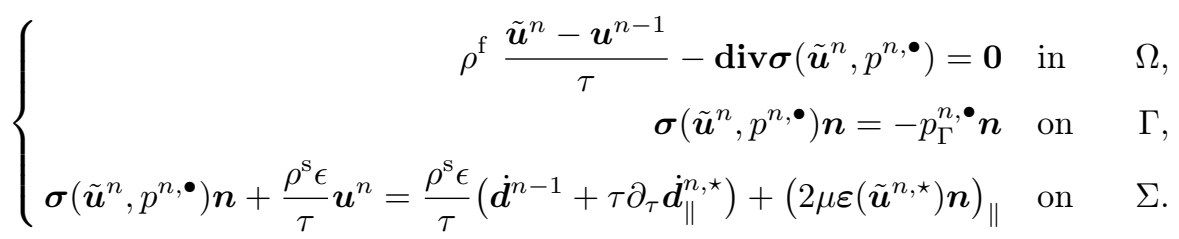

2. Fluid projection sub-step: find $\phi^{n}: \Omega \rightarrow \mathbb{R}$ such that

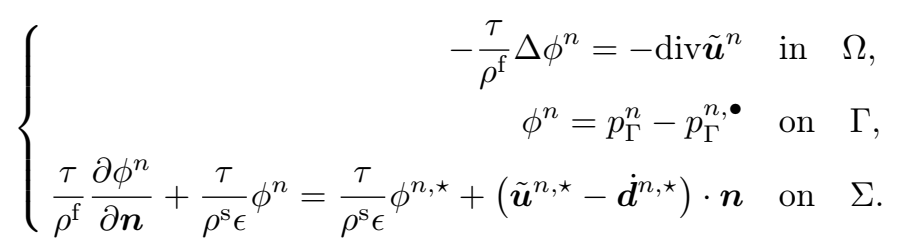

Thereafter set $p^{n}=\phi^{n}+p^{n, \bullet}, \boldsymbol{u}^{n}=\tilde{\boldsymbol{u}}^{n}-\frac{\tau}{\rho^{\mathrm{f}}} \boldsymbol{\nabla} \phi^{n}$.

3. Solid sub-step: find $\boldsymbol{d}^{n}: \Sigma \rightarrow \mathbb{R}^{3}$ and $\boldsymbol{\theta}^{n}: \Sigma \rightarrow \mathbb{R}^{3}$ with $\boldsymbol{\theta}_{\perp}^{n}=\mathbf{0}$ and such that

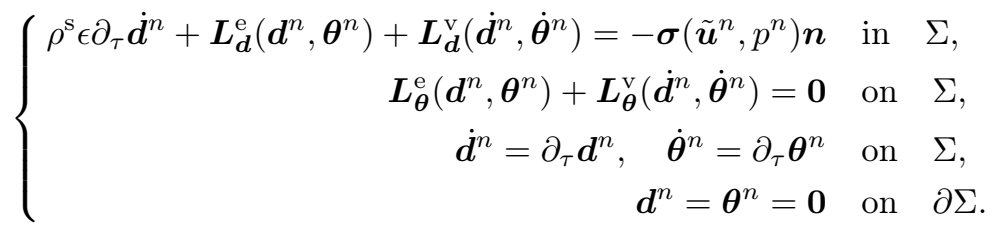

on $\Sigma$. These interface relations preserve the original splitting of (4) and (5) and enable the interface fluid-solid splitting through the explicit treatment of the solid viscoelastic terms in (7). Besides, the viscoelastic extrapolations are performed to control the perturbation of the kinematic coupling, which dramatically affects accuracy in practice (see Remark 7 and the numerical evidence of Section 4).

Remark 3 From (4), (5) and (7) we can observe that the extrapolations represented by the superscripts ${ }^{\bullet}$ and ${ }^{\star}$ are respectively associated to the velocity-pressure and fluid-solid splittings.

In summary, a three-stage splitting of the coupled problem (1)-(2) could be performed by solving (4) with $(7)_{1}$, then (5) with $(7)_{2}$ and finally (6). However, in order to avoid the extrapolations of the solid viscoelastic terms (which can be cumbersome in practice), we consider instead of (7) the following equivalent interface relations

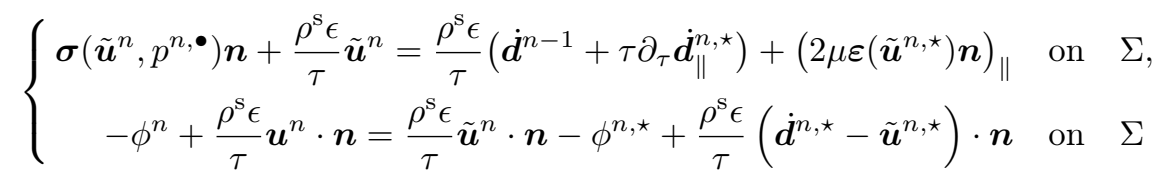

for $n>s+r$, whose derivation is detailed in the following. We first note that, from $(6)_{1}$, we 
have

$$
-\boldsymbol{L}_{\boldsymbol{d}}^{\mathrm{e}}\left(\boldsymbol{d}^{n, \star}, \boldsymbol{\theta}^{n, \star}\right)-\boldsymbol{L}_{\boldsymbol{d}}^{\mathrm{v}}\left(\dot{\boldsymbol{d}}^{n, \star}, \dot{\boldsymbol{\theta}}^{n, \star}\right)=\rho^{\mathrm{s}} \epsilon \partial_{\tau} \dot{\boldsymbol{d}}^{n, \star}+\boldsymbol{\sigma}\left(\tilde{\boldsymbol{u}}^{n, \star}, p^{n, \star}\right) \boldsymbol{n} \quad \text { on } \quad \Sigma
$$

for $n>r$. The tangential component of this expression writes

$$
-\boldsymbol{L}_{\boldsymbol{d}}^{\mathrm{e}}\left(\boldsymbol{d}^{n, \star}, \boldsymbol{\theta}^{n, \star}\right)_{\|}-\boldsymbol{L}_{\boldsymbol{d}}^{\mathrm{v}}\left(\dot{\boldsymbol{d}}^{n, \star}, \dot{\boldsymbol{\theta}}^{n, \star}\right)_{\|}=\rho^{\mathrm{s}} \epsilon \partial_{\tau} \dot{\boldsymbol{d}}_{\|}^{n, \star}+\left(2 \mu \varepsilon\left(\tilde{\boldsymbol{u}}^{n, \star}\right) \boldsymbol{n}\right)_{\|} \quad \text { on } \quad \Sigma,
$$

for $n>r$. Hence, by inserting this expression into $(7)_{1}$, we get $(11)_{1}$. We now proceed by taking the scalar product of (12) with $\boldsymbol{n}$, which yields

$$
-\boldsymbol{L}_{\boldsymbol{d}}^{\mathrm{e}}\left(\boldsymbol{d}^{n, \star}, \boldsymbol{\theta}^{n, \star}\right) \cdot \boldsymbol{n}-\boldsymbol{L}_{\boldsymbol{d}}^{\mathrm{v}}\left(\dot{\boldsymbol{d}}^{n, \star}, \dot{\boldsymbol{\theta}}^{n, \star}\right) \cdot \boldsymbol{n}
$$

$$
=\rho^{\mathrm{s}} \epsilon \partial_{\tau} \dot{\boldsymbol{d}}^{n, \star} \cdot \boldsymbol{n}+2 \mu \boldsymbol{n} \cdot \boldsymbol{\varepsilon}\left(\tilde{\boldsymbol{u}}^{n, \star}\right) \boldsymbol{n}-p^{n, \star} .
$$

In addition, by taking the scalar product of $(11)_{1}$ with $\boldsymbol{n}$, we get

$$
2 \mu \boldsymbol{n} \cdot \boldsymbol{\varepsilon}\left(\tilde{\boldsymbol{u}}^{n, \star}\right) \boldsymbol{n}=\left(p^{n, \bullet}\right)^{\star}-\frac{\rho^{\mathrm{s}} \epsilon}{\tau}\left(\tilde{\boldsymbol{u}}^{n, \star}-\dot{\boldsymbol{d}}^{n-1, \star}\right) \cdot \boldsymbol{n} \quad \text { on } \quad \Sigma
$$

for $n>s+r$. Hence, by inserting this expression into (13), it follows that

$$
\begin{aligned}
-\boldsymbol{L}_{\boldsymbol{d}}^{\mathrm{e}}\left(\boldsymbol{d}^{n, \star}, \boldsymbol{\theta}^{n, \star}\right) \cdot & \boldsymbol{n}-\boldsymbol{L}_{\boldsymbol{d}}^{\mathrm{v}}\left(\dot{\boldsymbol{d}}^{n, \star}, \dot{\boldsymbol{\theta}}^{n, \star}\right) \cdot \boldsymbol{n} \\
=\rho^{\mathrm{s}} \epsilon \partial_{\tau} \dot{\boldsymbol{d}}^{n, \star} \cdot \boldsymbol{n}-p^{n, \star}+\left(p^{n, \bullet}\right)^{\star}-\frac{\rho^{\mathrm{S}} \epsilon}{\tau}\left(\tilde{\boldsymbol{u}}^{n, \star}-\dot{\boldsymbol{d}}^{n-1, \star}\right) \cdot \boldsymbol{n} & =\left(p^{n, \bullet}\right)^{\star}-p^{n, \star}+\frac{\rho^{\mathrm{s}} \epsilon}{\tau}\left(\dot{\boldsymbol{d}}^{n, \star}-\tilde{\boldsymbol{u}}^{n, \star}\right) \cdot \boldsymbol{n}
\end{aligned}
$$

for $n>s+r$. We retrieve $(11)_{2}$ by inserting this last identity into $(7)_{2}$.

Finally, instead of the Darcy-step (5), we consider the equivalent pressure-Poisson formulation complemented with the following interface Robin condition, derived from $(11)_{2}$ :

$$
\frac{\tau}{\rho^{\mathrm{f}}} \frac{\partial \phi^{n}}{\partial \boldsymbol{n}}=-\left(\boldsymbol{u}^{n}-\tilde{\boldsymbol{u}}^{n}\right) \cdot \boldsymbol{n}=\frac{\tau}{\rho^{\mathrm{s}} \epsilon}\left(\phi^{n, \star}-\phi^{n}\right)+\left(\tilde{\boldsymbol{u}}^{n, \star}-\dot{\boldsymbol{d}}^{n, \star}\right) \cdot \boldsymbol{n} \quad \text { on } \quad \Sigma .
$$

The proposed fully decoupled schemes for problem (1)-(2) are reported in Algorithm 1. A salient feature of these methods is their intrinsic partitioned (or modular) character, in the sense that the overall fluid problem (8)-(9) does not depend on the specific structure of the solid model, and viceversa. In particular, the solid sub-step (10) is simply an implicit first-order time-discretization of the shell equations $(2)_{2-5}$ with a known forcing term. Note also that the interface Robin conditions $(8)_{3}$ and $(9)_{3}$ are nothing but consistent relaxations of the kinematic compatibility $(2)_{1}$. In this sense, it is worth recalling that for pressure-correction projection methods, Dirichlet conditions on the velocity yield homogeneous Neumann conditions for the pressure (see, e.g., $[40,41])$.

Remark 4 It should be noted that, in contrast to the approach recently proposed in [12], the Robin splitting provided by $(8)_{3}$ and $(9)_{3}$ admits a mathematically sound variational setting and, hence, can be straightforwardly combined with a finite element approximation in space (see Section 4).

Remark 5 For $s>1$ or $r>1$, Algorithm 1 is a multi-step method which requires initial data at the time instants $t_{1}, \ldots, t_{r+s}$. This additional data is obtained by performing $r+s$ steps of Algorithm 1 with lower values of $r$ or $s$, starting from $s=r=0$. For instance, if we consider Algorithm 1 with $s=1$ and $r=2$, we proceed as follows:

- one step of the scheme with $s=r=0$ to generate data at $t_{1}$;

- one step of the scheme with $s=1$ and $r=0$ (or $s=0$ and $r=1$ ) to generate data at $t_{2}$;

- one step of the scheme with $s=r=1$ to generate data at $t_{3}$. 


\subsection{Stability analysis}

In this section, energy estimates are derived for some of the schemes reported in Algorithm 1.

\subsubsection{Preliminaries}

Let $\omega$ be a given domain or surface in $\mathbb{R}^{3}$. The scalar product in $L^{2}(\omega)$ is denoted by $(\cdot, \cdot)_{\omega}$ and its norm by $\|\cdot\|_{\omega}$. For the sake of simplicity, the subscript $\Omega$ is omitted in the case $\omega=\Omega$. Since the elastic and viscous solid surface operators, $\left(\boldsymbol{L}_{\boldsymbol{d}}^{\mathrm{e}}, \boldsymbol{L}_{\boldsymbol{\theta}}^{\mathrm{e}}\right)$ and $\left(\boldsymbol{L}_{\boldsymbol{d}}^{\mathrm{v}}, \boldsymbol{L}_{\boldsymbol{\theta}}^{\mathrm{v}}\right)$, are supposed to be linear, they admit the following decomposition:

$$
\begin{array}{ll}
L_{d}^{\mathrm{e}}(d, \theta) \stackrel{\text { def }}{=} A_{d}^{\mathrm{e}} d+B_{d}^{\mathrm{e}} \theta, & L_{d}^{\mathrm{v}}(d, \theta) \stackrel{\text { def }}{=} A_{d}^{\mathrm{v}} d+B_{d}^{\mathrm{v}} \theta, \\
L_{\theta}^{\mathrm{e}}(d, \theta) \stackrel{\text { def }}{=} B_{\theta}^{\mathrm{e}} d+A_{\theta}^{\mathrm{e}} \theta, & L_{\theta}^{\mathrm{v}}(d, \theta) \stackrel{\text { def }}{=} B_{\theta}^{\mathrm{v}} d+A_{\theta}^{\mathrm{v}} \theta .
\end{array}
$$

Furthermore, we assume that the corresponding matrix operators

$$
\boldsymbol{Q}^{\mathrm{e}} \stackrel{\text { def }}{=}\left[\begin{array}{ll}
\boldsymbol{A}_{d}^{\mathrm{e}} & \boldsymbol{B}_{d}^{\mathrm{e}} \\
\boldsymbol{B}_{\theta}^{\mathrm{e}} & \boldsymbol{A}_{\theta}^{\mathrm{e}}
\end{array}\right], \quad \boldsymbol{Q}^{\mathrm{v}} \stackrel{\text { def }}{=}\left[\begin{array}{ll}
\boldsymbol{A}_{d}^{\mathrm{v}} & \boldsymbol{B}_{d}^{\mathrm{v}} \\
\boldsymbol{B}_{\theta}^{\mathrm{v}} & \boldsymbol{A}_{\theta}^{\mathrm{v}}
\end{array}\right],
$$

are self-adjoint positive definite operators in $\left[L^{2}(\Sigma)\right]^{3} \times\left[L^{2}(\Sigma)\right]^{3}$. An example of ReissnerMindling type shell model entering this abstract framework is given in A.

For the sake of conciseness, the following notation will also be used

$$
\boldsymbol{y} \stackrel{\text { def }}{=}\left[\begin{array}{l}
\boldsymbol{d} \\
\boldsymbol{\theta}
\end{array}\right], \quad \dot{\boldsymbol{y}} \stackrel{\text { def }}{=}\left[\begin{array}{l}
\dot{\boldsymbol{d}} \\
\dot{\boldsymbol{\theta}}
\end{array}\right],
$$

as well as their corresponding elastic-energy and viscous-dissipation norms

$$
\|\boldsymbol{y}\|_{\mathrm{e}} \stackrel{\text { def }}{=}\left(\boldsymbol{Q}^{\mathrm{e}} \boldsymbol{y}, \boldsymbol{y}\right)_{\Sigma}^{\frac{1}{2}}, \quad\|\dot{\boldsymbol{y}}\|_{\mathrm{v}} \stackrel{\text { def }}{=}\left(\boldsymbol{Q}^{\mathrm{v}} \dot{\boldsymbol{y}}, \dot{\boldsymbol{y}}\right)_{\Sigma}^{\frac{1}{2}} .
$$

In order to ease the presentation, we will commit a slight abuse of notation by settting $\boldsymbol{L}_{\boldsymbol{d}}^{\mathrm{e}} \boldsymbol{y} \stackrel{\text { def }}{=}$ $\boldsymbol{L}_{\boldsymbol{d}}^{\mathrm{e}}(\boldsymbol{d}, \boldsymbol{\theta})$ and $\boldsymbol{L}_{\boldsymbol{d}}^{\mathrm{v}} \dot{\boldsymbol{y}} \stackrel{\text { def }}{=} \boldsymbol{L}_{\boldsymbol{d}}^{\mathrm{v}}(\dot{\boldsymbol{d}}, \dot{\boldsymbol{\theta}})$. The same applies to operators $\boldsymbol{L}_{\boldsymbol{\theta}}^{\mathrm{e}}$ and $\boldsymbol{L}_{\boldsymbol{\theta}}^{\mathrm{v}}$.

\subsubsection{A priori energy estimates}

We define the time semi-discrete energy $E^{n}$ and dissipation $D^{n}$, at time $t_{n}$, as follows:

$$
\begin{aligned}
& E^{n} \stackrel{\text { def }}{=} \frac{\rho^{\mathrm{f}}}{2}\left\|\boldsymbol{u}^{n}\right\|^{2}+\frac{\rho^{\mathrm{s}} \epsilon}{2}\left\|\dot{\boldsymbol{d}}^{n}\right\|_{\Sigma}^{2}+\frac{1}{2}\left\|\boldsymbol{y}^{n}\right\|_{\mathrm{e}}^{2}+s \frac{\tau^{2}}{2 \rho^{\mathrm{f}}}\left\|\nabla p^{n}\right\|^{2}, \\
& D^{n} \stackrel{\text { def }}{=} 2 \mu\left\|\varepsilon\left(\tilde{\boldsymbol{u}}^{n}\right)\right\|^{2}+\left\|\dot{\boldsymbol{y}}^{n}\right\|_{\mathrm{v}}^{2}+(1-s) \frac{\tau}{2 \rho^{\mathrm{f}}}\left\|\boldsymbol{\nabla} p^{n}\right\|^{2} .
\end{aligned}
$$

The main result of this section is stated in the next theorem.

Theorem 1 Assume that the system is isolated, i.e., $p_{\Gamma}=0$ (free system) and let the sequence $\left\{\left(\tilde{\boldsymbol{u}}^{n}, \boldsymbol{u}^{n}, p^{n}, \boldsymbol{d}^{n}, \dot{\boldsymbol{d}}^{n}, \boldsymbol{\theta}^{n}, \dot{\boldsymbol{\theta}}^{n}\right)\right\}_{n>r}$ be given by Algorithm 1 either with $s=0$ and $r \in\{0,1\}$, or with $s=1$ and $r=0$. Then, the following a priori energy estimate holds for $n>s+r$ :

$$
E^{n}+\tau \sum_{m>s+r}^{n} D^{m} \leq E^{0} .
$$


Proof. We first reformulate the second step of Algorithm 1 as the following equivalent Darcy-problem:

$$
\left\{\begin{array}{rlll}
\rho^{\mathrm{f}} \frac{\boldsymbol{u}^{n}-\tilde{\boldsymbol{u}}^{n}}{\tau}+\boldsymbol{\nabla} \phi^{n}=\mathbf{0} & \text { in } & \Omega, \\
\operatorname{div} \boldsymbol{u}^{n}=0 & \text { in } & \Omega, \\
\phi^{n}=0 & \text { on } & \Gamma, \\
-\phi^{n}+\frac{\rho^{\mathrm{s}} \epsilon}{\tau} \boldsymbol{u}^{n} \cdot \boldsymbol{n}=\frac{\rho^{\mathrm{s}} \epsilon}{\tau} \tilde{\boldsymbol{u}}^{n} \cdot \boldsymbol{n}-\phi^{n, \star}+\frac{\rho^{\mathrm{s}} \epsilon}{\tau}\left(\dot{\boldsymbol{d}}^{n, \star}-\tilde{\boldsymbol{u}}^{n, \star}\right) \cdot \boldsymbol{n} & \text { on } & \Sigma .
\end{array}\right.
$$

Note that we have used the assumption that $p_{\Gamma}=0$. From $(8)_{3},(10)_{1}$ and $(16)_{4}$, it follows that (7) holds for $n>s+r$. Thus, by adding $(7)_{1}$ to $(7)_{2}$ multiplied by $\boldsymbol{n}$, we get

$$
\frac{\rho^{\mathrm{s}} \epsilon}{\tau}\left(\left(\tilde{\boldsymbol{u}}_{\|}^{n}+\boldsymbol{u}_{\perp}^{n}\right)-\dot{\boldsymbol{d}}^{n-1}\right)+\boldsymbol{L}_{\boldsymbol{d}}^{\mathrm{e}} \boldsymbol{y}^{n, \star}+\boldsymbol{L}_{\boldsymbol{d}}^{\mathrm{v}} \dot{\boldsymbol{y}}^{n, \star}=-\boldsymbol{\sigma}\left(\tilde{\boldsymbol{u}}^{n}, p^{n}\right) \boldsymbol{n} \quad \text { on } \quad \Sigma,
$$

which, after subtraction from $(10)_{1}$, yields the following fundamental displacement-velocity correction reformulation of the solid step:

$$
\frac{\rho^{\mathrm{s}} \epsilon}{\tau}\left(\dot{\boldsymbol{d}}^{n}-\left(\tilde{\boldsymbol{u}}_{\|}^{n}+\boldsymbol{u}_{\perp}^{n}\right)\right)+\boldsymbol{L}_{\boldsymbol{d}}^{\mathrm{e}}\left(\boldsymbol{y}^{n}-\boldsymbol{y}^{n, \star}\right)+\boldsymbol{L}_{\boldsymbol{d}}^{\mathrm{v}}\left(\dot{\boldsymbol{y}}^{n}-\dot{\boldsymbol{y}}^{n, \star}\right)=\mathbf{0} \quad \text { on } \quad \Sigma
$$

for $n>s+r$. As a result, from $(7)_{2}$ and (17), we obtain the following discrete kinematic relations on the interface $\Sigma$ :

$$
\left\{\begin{array}{r}
\boldsymbol{u}^{n} \cdot \boldsymbol{n}=\tilde{\boldsymbol{u}}^{n} \cdot \boldsymbol{n}+\frac{\tau}{\rho^{\mathrm{s}} \epsilon}\left(\phi^{n}-\boldsymbol{L}_{\boldsymbol{d}}^{\mathrm{e}} \boldsymbol{y}^{n, \star} \cdot \boldsymbol{n}-\boldsymbol{L}_{\boldsymbol{d}}^{\mathrm{v}} \dot{\boldsymbol{y}}^{n, \star} \cdot \boldsymbol{n}\right) \\
\tilde{\boldsymbol{u}}^{n}=\dot{\boldsymbol{d}}^{n}+\frac{\tau}{\rho^{\mathrm{s} \epsilon}}\left[\boldsymbol{L}_{\boldsymbol{d}}^{\mathrm{e}}\left(\boldsymbol{y}^{n}-\boldsymbol{y}^{n, \star}\right)+\boldsymbol{L}_{\boldsymbol{d}}^{\mathrm{v}}\left(\dot{\boldsymbol{y}}^{n}-\dot{\boldsymbol{y}}^{n, \star}\right)\right] \\
-\frac{\tau}{\rho^{\mathrm{s}} \epsilon}\left[\phi^{n} \boldsymbol{n}-\left(\boldsymbol{L}_{\boldsymbol{d}}^{\mathrm{e}} \boldsymbol{y}^{n, \star}\right)_{\perp}-\left(\boldsymbol{L}_{\boldsymbol{d}}^{\mathrm{v}} \dot{\boldsymbol{y}}^{n, \star}\right)_{\perp}\right]
\end{array}\right.
$$

for $n>s+r$. We now proceed by taking the scalar product of (8) $)_{1}$ and (16) 1 with $\tilde{\boldsymbol{u}}^{n}$ and $\boldsymbol{u}^{n}$, respectively. Hence, after integration by parts over $\Omega$, application of the boundary conditions $(8)_{2}$ and $(16)_{3}$, and summation of the resulting expressions, we get

$$
\begin{aligned}
\frac{\rho^{\mathrm{f}}}{2 \tau}\left(\left\|\boldsymbol{u}^{n}\right\|^{2}-\left\|\boldsymbol{u}^{n-1}\right\|^{2}+\left\|\boldsymbol{u}^{n}-\tilde{\boldsymbol{u}}^{n}\right\|^{2}\right)+2 \mu\left\|\varepsilon\left(\tilde{\boldsymbol{u}}^{n}\right)\right\|^{2} \\
-\left(\boldsymbol{\sigma}\left(\tilde{\boldsymbol{u}}^{n}, p^{n, \bullet}\right) \boldsymbol{n}, \tilde{\boldsymbol{u}}^{n}\right)_{\Sigma}-\left(p^{n, \bullet}, \operatorname{div} \tilde{\boldsymbol{u}}^{n}\right)+\left(\phi^{n}, \boldsymbol{u}^{n} \cdot \boldsymbol{n}\right)_{\Sigma} \leq 0 .
\end{aligned}
$$

Equivalently, by using $(16)_{1}$ and rearranging the interface terms, we obtain

$$
\begin{aligned}
& \frac{\rho^{\mathrm{f}}}{2 \tau}\left(\left\|\boldsymbol{u}^{n}\right\|^{2}-\left\|\boldsymbol{u}^{n-1}\right\|^{2}\right)+2 \mu\left\|\boldsymbol{\varepsilon}\left(\tilde{\boldsymbol{u}}^{n}\right)\right\|^{2}-\left(\boldsymbol{\sigma}\left(\tilde{\boldsymbol{u}}^{n}, p^{n}\right) \boldsymbol{n}, \tilde{\boldsymbol{u}}^{n}\right)_{\Sigma} \\
& +\left(p^{n},\left(\boldsymbol{u}^{n}-\tilde{\boldsymbol{u}}^{n}\right) \cdot \boldsymbol{n}\right)_{\Sigma}+\underbrace{\frac{\tau}{2 \rho^{\mathrm{f}}}\left\|\boldsymbol{\nabla} \phi^{n}\right\|^{2}-\left(p^{n, \bullet}, \operatorname{div} \tilde{\boldsymbol{u}}^{n}\right)-\left(p^{n, \bullet},\left(\boldsymbol{u}^{n}-\tilde{\boldsymbol{u}}^{n}\right) \cdot \boldsymbol{n}\right)_{\Sigma}}_{T_{1}} \leq 0 .
\end{aligned}
$$

The term $T_{1}$ can be controlled from $(16)_{1-3}$, using integration by parts, which yields

$$
\begin{aligned}
T_{1} & =\frac{\tau}{2 \rho^{\mathrm{f}}}\left\|\boldsymbol{\nabla} \phi^{n}\right\|^{2}-\frac{\tau}{\rho^{\mathrm{f}}}\left(\Delta \phi^{n}, p^{n, \bullet}\right)-\left(p^{n, \bullet},\left(\boldsymbol{u}^{n}-\tilde{\boldsymbol{u}}^{n}\right) \cdot \boldsymbol{n}\right)_{\Sigma} \\
& =\frac{\tau}{2 \rho^{\mathrm{f}}}\left\|\boldsymbol{\nabla} \phi^{n}\right\|^{2}+\frac{\tau}{\rho^{\mathrm{f}}}\left(\boldsymbol{\nabla} \phi^{n}, \boldsymbol{\nabla} p^{n, \bullet}\right)-\frac{\tau}{\rho^{\mathrm{f}}}\left(\frac{\partial \phi^{n}}{\partial \boldsymbol{n}}, p^{n, \bullet}\right)_{\Sigma}-\left(p^{n, \bullet},\left(\boldsymbol{u}^{n}-\tilde{\boldsymbol{u}}^{n}\right) \cdot \boldsymbol{n}\right)_{\Sigma} \\
& =\frac{\tau}{2 \rho^{\mathrm{f}}}\left(\left\|\boldsymbol{\nabla} p^{n}\right\|^{2}-\left\|\boldsymbol{\nabla} p^{n, \bullet}\right\|^{2}\right) .
\end{aligned}
$$


Thus, by inserting this expression into (19), we get

$$
\begin{aligned}
\frac{\rho^{\mathrm{f}}}{2 \tau}\left(\left\|\boldsymbol{u}^{n}\right\|^{2}-\left\|\boldsymbol{u}^{n-1}\right\|^{2}\right)+2 \mu\left\|\varepsilon\left(\tilde{\boldsymbol{u}}^{n}\right)\right\|^{2}+\frac{\tau}{2 \rho^{\mathrm{f}}} & \left(\left\|\boldsymbol{\nabla} p^{n}\right\|^{2}-\left\|\boldsymbol{\nabla} p^{n, \bullet}\right\|^{2}\right) \\
& -\left(\boldsymbol{\sigma}\left(\tilde{\boldsymbol{u}}^{n}, p^{n}\right) \boldsymbol{n}, \tilde{\boldsymbol{u}}^{n}\right)_{\Sigma}+\left(p^{n},\left(\boldsymbol{u}^{n}-\tilde{\boldsymbol{u}}^{n}\right) \cdot \boldsymbol{n}\right)_{\Sigma} \leq 0,
\end{aligned}
$$

which, owing to the relation $(18)_{1}$, yields

$$
\begin{aligned}
\frac{\rho^{\mathrm{f}}}{2 \tau}\left(\left\|\boldsymbol{u}^{n}\right\|^{2}-\left\|\boldsymbol{u}^{n-1}\right\|^{2}\right)+2 \mu\left\|\varepsilon\left(\tilde{\boldsymbol{u}}^{n}\right)\right\|^{2}+\frac{\tau}{2 \rho^{\mathrm{f}}}\left(\left\|\boldsymbol{\nabla} p^{n}\right\|^{2}-\left\|\boldsymbol{\nabla} p^{n, \bullet}\right\|^{2}\right) & \underbrace{-\left(\boldsymbol{\sigma}\left(\tilde{\boldsymbol{u}}^{n}, p^{n}\right) \boldsymbol{n}, \tilde{\boldsymbol{u}}^{n}\right)_{\Sigma}}_{T_{2}}+\frac{\tau}{\rho^{\mathrm{s}} \epsilon}\left(p^{n} \boldsymbol{n}, \phi^{n} \boldsymbol{n}-\left(\boldsymbol{L}_{\boldsymbol{d}}^{\mathrm{e}} \boldsymbol{y}^{n, \star}\right)_{\perp}-\left(\boldsymbol{L}_{\boldsymbol{d}}^{\mathrm{v}} \dot{\boldsymbol{y}}^{n, \star}\right)_{\perp}\right)_{\Sigma} \leq 0 .
\end{aligned}
$$

On the other hand, from $(10)_{1}$ and $(18)_{2}$, it follows that

$$
\begin{aligned}
T_{2}= & \frac{\rho^{\mathrm{s}} \epsilon}{2 \tau}\left(\left\|\dot{\boldsymbol{d}}^{n}\right\|_{\Sigma}^{2}-\left\|\dot{\boldsymbol{d}}^{n-1}\right\|_{\Sigma}^{2}+\left\|\dot{\boldsymbol{d}}^{n}-\dot{\boldsymbol{d}}^{n-1}\right\|_{\Sigma}^{2}\right)+\underbrace{\left(\boldsymbol{L}_{\boldsymbol{d}}^{\mathrm{e}} \boldsymbol{y}^{n}+\boldsymbol{L}_{\boldsymbol{d}}^{\mathrm{v}} \dot{\boldsymbol{y}}^{n}, \dot{\boldsymbol{d}}^{n}\right)_{\Sigma}}_{T_{2,1}} \\
& \left.+\frac{\tau}{2 \rho^{\mathrm{s} \epsilon}}\left(\left\|\boldsymbol{L}_{\boldsymbol{d}}^{\mathrm{e}} \boldsymbol{y}^{n}+\boldsymbol{L}_{\boldsymbol{d}}^{\mathrm{v}} \dot{\boldsymbol{y}}^{n}\right\|_{\Sigma}^{2}-\left\|\boldsymbol{L}_{\boldsymbol{d}}^{\mathrm{e}} \boldsymbol{y}^{n, \star}+\boldsymbol{L}_{\boldsymbol{d}}^{\mathrm{v}} \dot{\boldsymbol{y}}^{n, \star}\right\|_{\Sigma}^{2}\right)\right\}_{2,2} \\
& +\frac{\tau}{2 \rho^{\mathrm{s} \epsilon}}\left\|\boldsymbol{L}_{\boldsymbol{d}}^{\mathrm{e}}\left(\boldsymbol{y}^{n}-\boldsymbol{y}^{n, \star}\right)+\boldsymbol{L}_{\boldsymbol{d}}^{\mathrm{v}}\left(\dot{\boldsymbol{y}}^{n}-\dot{\boldsymbol{y}}^{n, \star}\right)\right\|_{\Sigma}^{2} \\
& +\underbrace{\left(\dot{\boldsymbol{d}}^{n}-\dot{\boldsymbol{d}}^{n-1}, \boldsymbol{L}_{\boldsymbol{d}}^{\mathrm{e}}\left(\boldsymbol{y}^{n}-\boldsymbol{y}^{n, \star}\right)+\boldsymbol{L}_{\boldsymbol{d}}^{\mathrm{v}}\left(\dot{\boldsymbol{y}}^{n}-\dot{\boldsymbol{y}}^{n, \star}\right)_{\Sigma}\right.}_{T_{2,3}} \\
& -\underbrace{\left(\dot{\boldsymbol{d}}^{n}-\dot{\boldsymbol{d}}^{n-1}, \phi^{n} \boldsymbol{n}-\left(\boldsymbol{L}_{\boldsymbol{d}}^{\mathrm{e}} \boldsymbol{y}^{n, \star}\right)_{\perp}-\left(\boldsymbol{L}_{\boldsymbol{d}}^{\mathrm{v}} \dot{\boldsymbol{y}}^{n, \star}\right)_{\perp}\right)_{\Sigma}}_{T_{2,4}} \\
& -\frac{\tau}{\rho^{\mathrm{s} \epsilon}}\left(\boldsymbol{L}_{\boldsymbol{d}}^{\mathrm{e}} \boldsymbol{y}^{n}+\boldsymbol{L}_{\boldsymbol{d}}^{\mathrm{v}} \dot{\boldsymbol{y}}^{n}, \phi^{n} \boldsymbol{n}-\left(\boldsymbol{L}_{\boldsymbol{d}}^{\mathrm{e}} \boldsymbol{y}^{n, \star}\right)_{\perp}-\left(\boldsymbol{L}_{\boldsymbol{d}}^{\mathrm{v}} \dot{\boldsymbol{y}}^{n, \star}\right)_{\perp}\right)_{\Sigma} \cdot
\end{aligned}
$$

In addition, thanks to $(10)_{2}$, for the term $T_{2,1}$ we have

$$
\begin{aligned}
T_{2,1} & =\left(\boldsymbol{L}_{\boldsymbol{d}}^{\mathrm{e}} \boldsymbol{y}^{n}+\boldsymbol{L}_{\boldsymbol{d}}^{\mathrm{v}} \dot{\boldsymbol{y}}^{n}, \dot{\boldsymbol{d}}^{n}\right)_{\Sigma}+\left(\boldsymbol{L}_{\boldsymbol{\theta}}^{\mathrm{e}} \boldsymbol{y}^{n}+\boldsymbol{L}_{\boldsymbol{\theta}}^{\mathrm{v}} \dot{\boldsymbol{y}}^{n}, \dot{\boldsymbol{\theta}}^{n}\right)_{\Sigma} \\
& =\left(\boldsymbol{Q}^{\mathrm{e}} \dot{\boldsymbol{y}}^{n}, \boldsymbol{y}^{n}\right)_{\Sigma}+\left(\boldsymbol{Q}^{\mathrm{v}} \dot{\boldsymbol{y}}^{n}, \dot{\boldsymbol{y}}^{n}\right)_{\Sigma} \\
& =\frac{1}{2 \tau}\left(\left\|\boldsymbol{y}^{n}\right\|_{\mathrm{e}}^{2}-\left\|\boldsymbol{y}^{n-1}\right\|_{\mathrm{e}}^{2}+\left\|\boldsymbol{y}^{n}-\boldsymbol{y}^{n-1}\right\|_{\mathrm{e}}^{2}\right)+\left\|\dot{\boldsymbol{y}}^{n}\right\|_{\mathrm{v}}^{2}
\end{aligned}
$$

Thus, by inserting this identity into (21) and the resulting expression into (20), we get the following energy inequality

$$
\begin{gathered}
\frac{\rho^{\mathrm{f}}}{2 \tau}\left(\left\|\boldsymbol{u}^{n}\right\|^{2}-\left\|\boldsymbol{u}^{n-1}\right\|^{2}\right)+\frac{\rho^{\mathrm{s}} \epsilon}{2 \tau}\left(\left\|\dot{\boldsymbol{d}}^{n}\right\|_{\Sigma}^{2}-\left\|\dot{\boldsymbol{d}}^{n-1}\right\|_{\Sigma}^{2}+\left\|\dot{\boldsymbol{d}}^{n}-\dot{\boldsymbol{d}}^{n-1}\right\|_{\Sigma}^{2}\right) \\
+\frac{1}{2 \tau}\left(\left\|\boldsymbol{y}^{n}\right\|_{\mathrm{e}}^{2}-\left\|\boldsymbol{y}^{n-1}\right\|_{\mathrm{e}}^{2}+\left\|\boldsymbol{y}^{n}-\boldsymbol{y}^{n-1}\right\|_{\mathrm{e}}^{2}\right)+\left\|\dot{\boldsymbol{y}}^{n}\right\|_{\mathrm{v}}^{2}+2 \mu\left\|\boldsymbol{\varepsilon}\left(\tilde{\boldsymbol{u}}^{n}\right)\right\|^{2} \\
+\frac{\tau}{2 \rho^{\mathrm{f}}}\left(\left\|\nabla p^{n}\right\|^{2}-\left\|\boldsymbol{\nabla} p^{n, \bullet}\right\|^{2}\right)+T_{2,2}+T_{2,3}+T_{2,4} \\
+\underbrace{\frac{\tau}{\rho^{\mathrm{s}} \epsilon}\left(p^{n} \boldsymbol{n}-\boldsymbol{L}_{\boldsymbol{d}}^{\mathrm{e}} \boldsymbol{y}^{n}-\boldsymbol{L}_{\boldsymbol{d}}^{\mathrm{v}} \dot{\boldsymbol{y}}^{n}, \phi^{n} \boldsymbol{n}-\left(\boldsymbol{L}_{\boldsymbol{d}}^{\mathrm{e}} \boldsymbol{y}^{n, \star}\right)_{\perp}-\left(\boldsymbol{L}_{\boldsymbol{d}}^{\mathrm{v}} \dot{\boldsymbol{y}}^{n, \star}\right)_{\perp}\right)_{\Sigma} \leq 0}_{T_{3}} \leq 0
\end{gathered}
$$

$\mathrm{RR} \mathrm{n}^{\circ} 8425$ 
for $n>s+r$.

We now proceed by treating each case of extrapolation separately. Algorithm 1 with $s \in\{0,1\}$ and $r=0$. We have

$$
\begin{aligned}
T_{2,3}+T_{2,4} \geq & -\frac{\rho^{\mathrm{s}} \epsilon}{2 \tau}\left\|\dot{\boldsymbol{d}}^{n}-\dot{\boldsymbol{d}}^{n-1}\right\|_{\Sigma}^{2}-\frac{\tau}{2 \rho^{\mathrm{s}} \epsilon}\left\|\boldsymbol{L}_{\boldsymbol{d}}^{\mathrm{e}} \boldsymbol{y}^{n}+\boldsymbol{L}_{\boldsymbol{d}}^{\mathrm{v}} \dot{\boldsymbol{y}}^{n}-\phi^{n} \boldsymbol{n}\right\|_{\Sigma} \\
T_{2,2}+T_{3}= & \frac{\tau}{2 \rho^{\mathrm{s}} \epsilon}\left(\left\|p^{n}\right\|_{\Sigma}^{2}-\left\|p^{n, \bullet}\right\|_{\Sigma}^{2}\right)+\frac{\tau}{2 \rho^{\mathrm{s}} \epsilon}\left\|\boldsymbol{L}_{\boldsymbol{d}}^{\mathrm{e}} \boldsymbol{y}^{n}+\boldsymbol{L}_{\boldsymbol{d}}^{\mathrm{v}} \dot{\boldsymbol{y}}^{n}-\phi^{n} \boldsymbol{n}\right\|_{\Sigma}^{2} \\
& +\frac{\tau}{2 \rho^{\mathrm{s}} \epsilon}\left\|\boldsymbol{L}_{\boldsymbol{d}}^{\mathrm{e}} \boldsymbol{y}^{n}+\boldsymbol{L}_{\boldsymbol{d}}^{\mathrm{v}} \dot{\boldsymbol{y}}^{n}\right\|_{\Sigma}^{2}
\end{aligned}
$$

Therefore,

$$
\begin{aligned}
T_{2,2}+T_{2,3}+T_{2,4}+T_{3} \geq-\frac{\rho^{\mathrm{s}} \epsilon}{2 \tau}\left\|\dot{\boldsymbol{d}}^{n}-\dot{\boldsymbol{d}}^{n-1}\right\|_{\Sigma}^{2}+\frac{\tau}{2 \rho^{\mathrm{s}} \epsilon}\left(\left\|p^{n}\right\|_{\Sigma}^{2}-\left\|p^{n, \bullet}\right\|_{\Sigma}^{2}\right) & +\frac{\tau}{2 \rho^{\mathrm{s}} \epsilon}\left\|\boldsymbol{L}_{\boldsymbol{d}}^{\mathrm{e}} \boldsymbol{y}^{n}+\boldsymbol{L}_{\boldsymbol{d}}^{\mathrm{v}} \dot{\boldsymbol{y}}^{n}\right\|_{\Sigma}^{2} .
\end{aligned}
$$

For $s=0$, the estimate (15) follows by inserting this expression into (23), multiplication by $\tau$ and summation over $m=1, \ldots, n$. For $s=1$, we can only sum over $m=2, \ldots, n$, which yields

$$
E^{n}+\tau \sum_{m=2}^{n} D^{m} \leq E^{1}+\frac{\tau^{2}}{2 \rho^{\mathrm{s}} \epsilon}\left\|p^{1}\right\|_{\Sigma}^{2}
$$

Since $p^{1}$ is generated with one step of the scheme with $s=r=0$ (see Remark 5), the last term of (25) can be controlled by the extra dissipation provided by (24) with $s=0$ and $n=1$. As a result, estimate (15) also holds for $s=1$ and $r=0$.

Algorithm 1 with $s=0$ and $r=1$. From $(10)_{2}$, we have

$$
\begin{aligned}
T_{2,3} & =\tau^{2}\left(\partial_{\tau} \dot{\boldsymbol{d}}^{n}, \boldsymbol{L}_{\boldsymbol{d}}^{\mathrm{e}} \dot{\boldsymbol{y}}^{n}+\boldsymbol{L}_{\boldsymbol{d}}^{\mathrm{v}}\left(\partial_{\tau} \dot{\boldsymbol{y}}^{n}\right)\right)_{\Sigma}+\tau^{2}\left(\partial_{\tau} \dot{\boldsymbol{\theta}}^{n}, \boldsymbol{L}_{\boldsymbol{\theta}}^{\mathrm{e}} \dot{\boldsymbol{y}}^{n}+\boldsymbol{L}_{\boldsymbol{\theta}}^{\mathrm{v}}\left(\partial_{\tau} \dot{\boldsymbol{y}}^{n}\right)\right)_{\Sigma} \\
& =\tau^{2}\left(\boldsymbol{Q}^{\mathrm{e}} \dot{\boldsymbol{y}}^{n}, \partial_{\tau} \dot{\boldsymbol{y}}^{n}\right)_{\Sigma}+\tau^{2}\left(\boldsymbol{Q}^{\mathrm{v}} \partial_{\tau} \dot{\boldsymbol{y}}^{n}, \partial_{\tau} \dot{\boldsymbol{y}}^{n}\right)_{\Sigma} \\
& =\frac{\tau}{2}\left(\left\|\dot{\boldsymbol{y}}^{n}\right\|_{\mathrm{e}}^{2}-\left\|\dot{\boldsymbol{y}}^{n-1}\right\|_{\mathrm{e}}^{2}+\left\|\dot{\boldsymbol{y}}^{n}-\dot{\boldsymbol{y}}^{n-1}\right\|_{\mathrm{e}}^{2}\right)+\tau^{2}\left\|\partial_{\tau} \dot{\boldsymbol{y}}^{n}\right\|_{\mathrm{v}}^{2}
\end{aligned}
$$

for $n \geq 2$. For the third term, we get

$$
T_{2,4} \geq-\frac{\rho^{\mathrm{s}} \epsilon}{2 \tau}\left\|\dot{\boldsymbol{d}}^{n}-\dot{\boldsymbol{d}}^{n-1}\right\|_{\Sigma}^{2}-\frac{\tau}{2 \rho^{\mathrm{s}} \epsilon}\left\|\left(\boldsymbol{L}_{\boldsymbol{d}}^{\mathrm{e}} \boldsymbol{y}^{n-1}\right)_{\perp}+\left(\boldsymbol{L}_{\boldsymbol{d}}^{\mathrm{v}} \dot{\boldsymbol{y}}^{n-1}\right)_{\perp}-p^{n} \boldsymbol{n}\right\|_{\Sigma}^{2}
$$

At last, the fourth term is estimated as follows

$$
\begin{aligned}
T_{3}= & \frac{\tau}{\rho^{\mathrm{s}} \epsilon}\left(p^{n} \boldsymbol{n}-\boldsymbol{L}_{\boldsymbol{d}}^{\mathrm{e}} \boldsymbol{y}^{n}-\boldsymbol{L}_{\boldsymbol{d}}^{\mathrm{v}} \dot{\boldsymbol{y}}^{n}, p^{n} \boldsymbol{n}-\left(\boldsymbol{L}_{\boldsymbol{d}}^{\mathrm{e}} \boldsymbol{y}^{n-1}\right)_{\perp}-\left(\boldsymbol{L}_{\boldsymbol{d}}^{\mathrm{v}} \dot{\boldsymbol{y}}^{n-1}\right)_{\perp}\right)_{\Sigma} \\
= & \frac{\tau}{\rho^{\mathrm{s}} \epsilon}\left(p^{n} \boldsymbol{n}-\left(\boldsymbol{L}_{\boldsymbol{d}}^{\mathrm{e}} \boldsymbol{y}^{n}\right)_{\perp}-\left(\boldsymbol{L}_{\boldsymbol{d}}^{\mathrm{v}} \dot{\boldsymbol{y}}^{n}\right)_{\perp}, p^{n} \boldsymbol{n}-\left(\boldsymbol{L}_{\boldsymbol{d}}^{\mathrm{e}} \boldsymbol{y}^{n-1}\right)_{\perp}-\left(\boldsymbol{L}_{\boldsymbol{d}}^{\mathrm{v}} \dot{\boldsymbol{y}}^{n-1}\right)_{\perp}\right)_{\Sigma} \\
= & \frac{\tau}{\rho^{\mathrm{s} \epsilon}}\left\|p^{n} \boldsymbol{n}-\left(\boldsymbol{L}_{\boldsymbol{d}}^{\mathrm{e}} \boldsymbol{y}^{n-1}\right)_{\perp}-\left(\boldsymbol{L}_{\boldsymbol{d}}^{\mathrm{v}} \dot{\boldsymbol{y}}^{n-1}\right)_{\perp}\right\|_{\Sigma}^{2} \\
& -\frac{\tau}{\rho^{\mathrm{s}} \epsilon}\left(\left(\boldsymbol{L}_{\boldsymbol{d}}^{\mathrm{e}}\left(\boldsymbol{y}^{n}-\boldsymbol{y}^{n-1}\right)\right)_{\perp}+\left(\boldsymbol{L}_{\boldsymbol{d}}^{\mathrm{v}}\left(\dot{\boldsymbol{y}}^{n}-\dot{\boldsymbol{y}}^{n-1}\right)\right)_{\perp}, p^{n} \boldsymbol{n}-\left(\boldsymbol{L}_{\boldsymbol{d}}^{\mathrm{e}} \boldsymbol{y}^{n-1}\right)_{\perp}-\left(\boldsymbol{L}_{\boldsymbol{d}}^{\mathrm{v}} \dot{\boldsymbol{y}}^{n-1}\right)_{\perp}\right)_{\Sigma} \\
\geq & \frac{\tau}{2 \rho^{\mathrm{s} \epsilon}}\left\|p^{n} \boldsymbol{n}-\left(\boldsymbol{L}_{\boldsymbol{d}}^{\mathrm{e}} \boldsymbol{y}^{n-1}\right)_{\perp}-\left(\boldsymbol{L}_{\boldsymbol{d}}^{\mathrm{v}} \dot{\boldsymbol{y}}^{n-1}\right)_{\perp}\right\|_{\Sigma}^{2} \\
& -\frac{\tau}{2 \rho^{\mathrm{s}} \epsilon}\left\|\left(\boldsymbol{L}_{\boldsymbol{d}}^{\mathrm{e}}\left(\boldsymbol{y}^{n}-\boldsymbol{y}^{n-1}\right)\right)_{\perp}+\left(\boldsymbol{L}_{\boldsymbol{d}}^{\mathrm{v}}\left(\dot{\boldsymbol{y}}^{n}-\dot{\boldsymbol{y}}^{n-1}\right)\right)_{\perp}\right\|_{\Sigma}^{2} .
\end{aligned}
$$


Therefore, by collecting the above estimations, we get

$$
\begin{aligned}
T_{2,2}+T_{2,3}+T_{2,4}+ & T_{3} \geq-\frac{\rho^{\mathrm{s}} \epsilon}{2 \tau}\left\|\dot{\boldsymbol{d}}^{n}-\dot{\boldsymbol{d}}^{n-1}\right\|_{\Sigma}^{2} \\
+ & \frac{\tau}{2}\left(\left\|\dot{\boldsymbol{y}}^{n}\right\|_{\mathrm{e}}^{2}-\left\|\dot{\boldsymbol{y}}^{n-1}\right\|_{\mathrm{e}}^{2}+\left\|\dot{\boldsymbol{y}}^{n}-\dot{\boldsymbol{y}}^{n-1}\right\|_{\mathrm{e}}^{2}\right)+\tau^{2}\left\|\partial_{\tau} \dot{\boldsymbol{y}}^{n}\right\|_{\mathrm{v}}^{2} \\
& +\frac{\tau}{2 \rho^{\mathrm{s}} \epsilon}\left(\left\|\boldsymbol{L}_{\boldsymbol{d}}^{\mathrm{e}} \boldsymbol{y}^{n}+\boldsymbol{L}_{\boldsymbol{d}}^{\mathrm{v}} \dot{\boldsymbol{y}}^{n}\right\|_{\Sigma}^{2}-\left\|\boldsymbol{L}_{\boldsymbol{d}}^{\mathrm{e}} \boldsymbol{y}^{n-1}+\boldsymbol{L}_{\boldsymbol{d}}^{\mathrm{v}} \dot{\boldsymbol{y}}^{n-1}\right\|_{\Sigma}^{2}\right) \\
& +\frac{\tau}{2 \rho^{\mathrm{s}} \epsilon}\left\|\left(\boldsymbol{L}_{\boldsymbol{d}}^{\mathrm{e}}\left(\boldsymbol{y}^{n}-\boldsymbol{y}^{n-1}\right)\right)_{\|}+\left(\boldsymbol{L}_{\boldsymbol{d}}^{\mathrm{v}}\left(\dot{\boldsymbol{y}}^{n}-\dot{\boldsymbol{y}}^{n-1}\right)\right)_{\|}\right\|_{\Sigma}^{2} .
\end{aligned}
$$

Inserting this expression into (23), then multiplying by $\tau$ and summing over $m=2, \ldots, n$, yields the estimate

$$
E^{n}+\tau \sum_{m=2}^{n} D^{m} \leq E^{1}+\frac{\tau}{2}\left\|\boldsymbol{y}^{1}-\boldsymbol{y}^{0}\right\|_{\mathrm{e}}^{2}+\frac{\tau^{2}}{2 \rho^{\mathrm{s}} \epsilon}\left\|\boldsymbol{L}_{\boldsymbol{d}}^{\mathrm{e}} \boldsymbol{y}^{1}+\boldsymbol{L}_{\boldsymbol{d}}^{\mathrm{v}} \dot{\boldsymbol{y}}^{1}\right\|_{\Sigma}^{2}
$$

Owing to the initialization procedure (see Remark 5), $\boldsymbol{y}^{1}$ and $\dot{\boldsymbol{y}}^{1}$ are obtained from the scheme with $s=r=0$. Hence, the right-hand side of (26) can be bounded from (15), with $s=r=0$, and the numerical dissipation provided by (22) and (24) for $n=1$. This completes the proof.

Theorem 1 guarantees the unconditionally energy stability of Algorithm 1 with:

- $s=0$ (non-incremental pressure-correction) and $r=0,1$;

- $s=1$ (incremental pressure-correction) and $r=0$.

Similar energy estimates were obtained in [36, Theorem 1] for the original Robin-Neumann schemes with a monolithic time-stepping in the fluid and a simpler solid model. This indicates that, at least for the above variants, the extensions proposed in this work preserve their stability properties.

Remark 6 The stability of Algorithm 1 with $s=0$ and $r=2$, or $s=1$ and $r \in\{1,2\}$, is not covered by the previous analysis. In fact, it appears that the arguments used in the proof above can not be straightforwardly adapted to cope with these variants. Numerical evidence, reported in Section 4, indicates however that these schemes deliver stable and accurate numerical approximations.

Remark 7 From (17) it follows that

$$
\tilde{\boldsymbol{u}}_{\|}^{n}+\boldsymbol{u}_{\perp}^{n}=\dot{\boldsymbol{d}}^{n}+\frac{\tau}{\rho^{\mathrm{s}} \epsilon}\left[\boldsymbol{L}_{\boldsymbol{d}}^{\mathrm{e}}\left(\boldsymbol{y}^{n}-\boldsymbol{y}^{n, \star}\right)+\boldsymbol{L}_{\boldsymbol{d}}^{\mathrm{v}}\left(\dot{\boldsymbol{y}}^{n}-\dot{\boldsymbol{y}}^{n, \star}\right)\right] \quad \text { on } \quad \Sigma .
$$

Hence, the kinematic constraint (2) in Algorithm 1 is enforced in terms of the tangent, $\tilde{\boldsymbol{u}}_{\|}^{n}$, and normal components, $\boldsymbol{u}_{\perp}^{n}$, of the intermediate and end-of-step fluid velocities, respectively. Note that the consistency of (27) is given by the order $r \in\{0,1,2\}$ of the explicit extrapolations on the interface, irrespectively of the non-incremental $(s=0)$ or incremental $(s=1)$ nature of the projection method in the fluid. This indicates that the accuracy of the fluid-solid splitting induced by Algorithm 1 is dictated by $r \in\{0,1,2\}$, while $s \in\{0,1\}$ drives the accuracy of the time-marching in the fluid.

Remark 8 Besides the technical difficulties introduced by the projection method in the analysis of Algorithm 1, the result of Theorem 1 makes a step forward with respect to the stability analyses of [31, 36], where the solid model is assumed to be simply a membrane or a Koiter type shell (see also [42, 17, 53, 12]). It is also worth recalling that, in contrast to Theorem 1, the stability result recently reported in [12] relies on modal analysis (in further simplified models and geometrical configurations) and does not cover velocity/pressure splitting in the fluid. 


\section{The non-linear case}

In this section, the splitting schemes reported in Algorithm 1 are formulated in a non-linear framework. The fluid is modeled by the incompressible Navier-Stokes equations in ALE (arbitrary Lagrangian-Eulerian) formulation (see, e.g. [27, 37]). The structure is described by a non-linear Reissner-Mindlin shell model (see, e.g., [21, 13]). The fluid domain motion is parametrized by

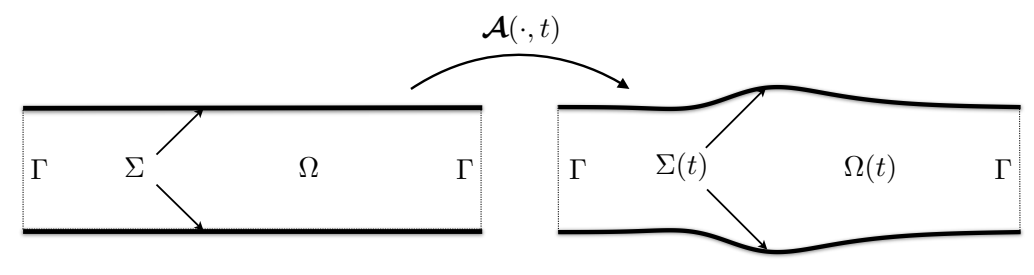

Figure 1: Geometrical configurations.

the ALE map $\mathcal{A} \stackrel{\text { def }}{=} \boldsymbol{I}_{\Omega}+\boldsymbol{d}^{\mathrm{f}}$, where $\boldsymbol{d}^{\mathrm{f}}: \Omega \times \mathbb{R}^{+} \rightarrow \mathbb{R}^{3}$ stands for the fluid domain displacement (see Figure 1). Hence, we have $\Omega(t)=\mathcal{A}(\Omega, t)$. The ALE time derivative operator is denoted by $\left.\partial_{t}\right|_{\mathcal{A}}$. The symbol $\boldsymbol{w} \stackrel{\text { def }}{=} \partial_{t} \mathcal{A}=\partial_{t} \boldsymbol{d}^{\mathrm{f}}$ stands for the fluid domain velocity, $\boldsymbol{F} \stackrel{\text { def }}{=} \boldsymbol{\nabla} \mathcal{A}$ for the gradient of deformation and $J \stackrel{\text { def }}{=} \operatorname{det} \boldsymbol{F}$ for the Jacobian.

The considered non-linear fluid-structure problem reads as follows: find the fluid domain displacement $\boldsymbol{d}^{\mathrm{f}}: \Omega \times \mathbb{R}^{+} \rightarrow \mathbb{R}^{3}$, the velocity $\boldsymbol{u}: \Omega \times \mathbb{R}^{+} \rightarrow \mathbb{R}^{3}$, the pressure $p: \Omega \times \mathbb{R}^{+} \rightarrow \mathbb{R}$, the solid mid-surface displacement $\boldsymbol{d}: \Sigma \times \mathbb{R}^{+} \rightarrow \mathbb{R}^{3}$ and the rotation vector $\boldsymbol{\theta}: \Sigma \times \mathbb{R}^{+} \rightarrow \mathbb{R}^{3}$, with $\boldsymbol{\theta}_{\perp}=\mathbf{0}$, such that

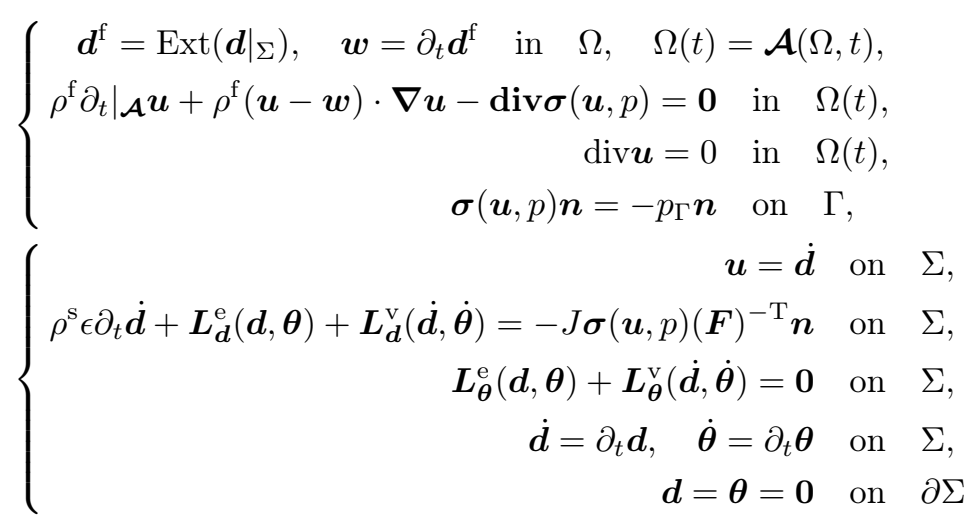

and fulfilling the initial conditions $\boldsymbol{u}(0)=\boldsymbol{u}_{0}, \boldsymbol{d}(0)=\boldsymbol{d}_{0}, \boldsymbol{\theta}(0)=\boldsymbol{\theta}_{0}$ and $\dot{\boldsymbol{d}}(0)=\dot{\boldsymbol{d}}_{0}$. The symbol $\operatorname{Ext}(\cdot)$ represents an arbitrary extension operator (e.g., an harmonic lifting) from the interface $\Sigma$ into the fluid domain $\Omega$ and which vanishes on $\Gamma$. At last, the surface differential operators $\left(\boldsymbol{L}_{\boldsymbol{d}}^{\mathrm{e}}, \boldsymbol{L}_{\boldsymbol{\theta}}^{\mathrm{e}}\right)$ and $\left(\boldsymbol{L}_{\boldsymbol{d}}^{\mathrm{v}}, \boldsymbol{L}_{\boldsymbol{\theta}}^{\mathrm{v}}\right)$ describe the (possibly non-linear) shell elastic and viscous contributions, respectively.

Remark 9 Note that the physical quantities $\boldsymbol{u}, p$ and $\boldsymbol{w}$ are defined in the reference fluid domain $\Omega$. In (28) they are evaluated in the current fluid domain, $\Omega(t)$, by composition with $\mathcal{A}^{-1}(\cdot, t)$. In order to ease the presentation, this change of variable is not specified in the equations. 
Algorithm 2 Fully decoupled schemes for (28)-(29).

For $n>s+r$ :

1. Fluid domain update:

$$
\boldsymbol{d}^{\mathrm{f}, n}=\operatorname{Ext}\left(\left.\boldsymbol{d}^{n-1}\right|_{\Sigma}\right), \quad \boldsymbol{w}^{n}=\partial_{\tau} \boldsymbol{d}^{\mathrm{f}, n}, \quad \mathcal{A}^{n}=\boldsymbol{I}_{\Omega}+\boldsymbol{d}^{\mathrm{f}, n}, \quad \Omega^{n}=\mathcal{A}^{n}(\Omega)
$$

and then set $\boldsymbol{F}^{n}=\nabla \mathcal{A}^{n}, J^{n}=\operatorname{det} \boldsymbol{F}^{n}$.

2. Fluid viscous sub-step: find $\tilde{\boldsymbol{u}}^{n}: \Omega \rightarrow \mathbb{R}^{3}$ such that

$$
\left\{\begin{array}{rrrr}
\left.\rho^{\mathrm{f}} \frac{\tilde{\boldsymbol{u}}^{n}-\boldsymbol{u}^{n-1}}{\tau}\right|_{\mathcal{A}}+\rho^{\mathrm{f}}\left(\tilde{\boldsymbol{u}}^{n-1}-\boldsymbol{w}^{n}\right) \cdot \boldsymbol{\nabla} \tilde{\boldsymbol{u}}^{n}-\operatorname{div} \boldsymbol{\sigma}\left(\tilde{\boldsymbol{u}}^{n}, p^{n, \bullet}\right)=\mathbf{0} & \text { in } & \Omega^{n}, \\
\boldsymbol{\sigma}\left(\tilde{\boldsymbol{u}}^{n}, p^{n, \bullet}\right) \boldsymbol{n}=\mathbf{0} & \text { on } & \Gamma, \\
J^{n} \boldsymbol{\sigma}\left(\boldsymbol{u}^{n}, p^{n, \bullet}\right)\left(\boldsymbol{F}^{n}\right)^{-\mathrm{T}} \boldsymbol{n}+\frac{\rho^{\mathrm{s}} \epsilon}{\tau} \boldsymbol{u}^{n}=\frac{\rho^{\mathrm{S}} \epsilon}{\tau}\left(\dot{\boldsymbol{d}}^{n-1}+\tau \partial_{\tau} \dot{\boldsymbol{d}}_{\|}^{n, \star}\right) & & \\
& +2 \mu\left(J \boldsymbol{\varepsilon}(\tilde{\boldsymbol{u}}) \boldsymbol{F}^{-\mathrm{T}} \boldsymbol{n}\right)_{\|}^{n, \star} & \text { on } & \Sigma .
\end{array}\right.
$$

3. Fluid projection sub-step: find $\phi^{n}: \Omega \rightarrow \mathbb{R}$ such that

$$
\left\{\begin{array}{r}
-\frac{\tau}{\rho^{\mathrm{f}}} \Delta \phi^{n}=-\operatorname{div} \tilde{\boldsymbol{u}}^{n} \quad \text { in } \quad \Omega^{n}, \\
\phi^{n}=p_{\Gamma}\left(t_{n}\right)-p^{n, \bullet} \quad \text { on } \quad \Gamma \\
\frac{\tau}{\rho^{\mathrm{f}}} \frac{\partial \phi^{n}}{\partial \boldsymbol{n}}+\frac{\tau}{\rho^{\mathrm{s}} \epsilon} \phi^{n}=\frac{\tau}{\rho^{\mathrm{s}} \epsilon} \phi^{n, \star}+\left(\tilde{\boldsymbol{u}}^{n, \star}-\dot{\boldsymbol{d}}^{n, \star}\right) \cdot \boldsymbol{n} \quad \text { on } \quad \Sigma .
\end{array}\right.
$$

Thereafter set $p^{n}=\phi^{n}+p^{n, \bullet}, \boldsymbol{u}^{n}=\tilde{\boldsymbol{u}}^{n}-\frac{\tau}{\rho^{\mathrm{f}}} \boldsymbol{\nabla} \phi^{n}$ in $\Omega$.

4. Solid sub-step: find $\boldsymbol{d}^{n}: \Sigma \rightarrow \mathbb{R}^{3}$ and $\boldsymbol{\theta}^{n}: \Sigma \rightarrow \mathbb{R}^{3}$ with $\boldsymbol{\theta}_{\perp}^{n}=\mathbf{0}$, such that

$$
\left\{\begin{array}{rccc}
\rho^{\mathrm{s}} \epsilon \partial_{\tau} \dot{\boldsymbol{d}}^{n}+\boldsymbol{L}_{\boldsymbol{d}}^{\mathrm{e}}\left(\boldsymbol{d}^{n}, \boldsymbol{\theta}^{n}\right)+\boldsymbol{L}_{\boldsymbol{d}}^{\mathrm{v}}\left(\dot{\boldsymbol{d}}^{n}, \dot{\boldsymbol{\theta}}^{n}\right)=-J^{n} \boldsymbol{\sigma}\left(\tilde{\boldsymbol{u}}^{n}, p^{n}\right)\left(\boldsymbol{F}^{n}\right)^{-\mathrm{T}} \boldsymbol{n} & \text { on } & \Sigma, \\
\boldsymbol{L}_{\boldsymbol{\theta}}^{\mathrm{e}}\left(\boldsymbol{d}^{n}, \boldsymbol{\theta}^{n}\right)+\boldsymbol{L}_{\boldsymbol{\theta}}^{\mathrm{v}}\left(\dot{\boldsymbol{d}}^{n}, \dot{\boldsymbol{\theta}}^{n}\right)=\mathbf{0} & \text { on } & \Sigma, \\
\dot{\boldsymbol{d}}^{n}=\partial_{\tau} \boldsymbol{d}^{n}, \quad \dot{\boldsymbol{\theta}}^{n}=\partial_{\tau} \boldsymbol{\theta}^{n} & \text { on } & \Sigma, \\
\boldsymbol{d}^{n}=\boldsymbol{\theta}^{n}=\mathbf{0} & \text { on } & \partial \Sigma .
\end{array}\right.
$$

Remark 10 A simple expression for the viscous operator $\left(\boldsymbol{L}_{\boldsymbol{d}}^{\mathrm{v}}, \boldsymbol{L}_{\boldsymbol{\theta}}^{\mathrm{v}}\right)$ is given by the generalized Rayleigh relation:

$$
\boldsymbol{L}_{\boldsymbol{d}}^{\mathrm{v}}(\dot{\boldsymbol{d}}, \dot{\boldsymbol{\theta}})=\alpha_{0} \rho^{\mathrm{s}} \epsilon \dot{\boldsymbol{d}}+\alpha_{1} D \boldsymbol{L}_{\boldsymbol{d}}^{\mathrm{e}}(\boldsymbol{d}, \boldsymbol{\theta})(\dot{\boldsymbol{d}}, \dot{\boldsymbol{\theta}}), \quad \boldsymbol{L}_{\boldsymbol{\theta}}^{\mathrm{v}}(\dot{\boldsymbol{d}}, \dot{\boldsymbol{\theta}})=\alpha_{1} D \boldsymbol{L}_{\boldsymbol{\theta}}^{\mathrm{e}}(\boldsymbol{d}, \boldsymbol{\theta})(\dot{\boldsymbol{d}}, \dot{\boldsymbol{\theta}}) .
$$

Here, $\alpha_{0}, \alpha_{1}>0$ are given parameters and $D \boldsymbol{L}_{\boldsymbol{d}}^{\mathrm{e}}(\boldsymbol{d}, \boldsymbol{\theta}), D \boldsymbol{L}_{\boldsymbol{\theta}}^{\mathrm{e}}(\boldsymbol{d}, \boldsymbol{\theta})$ denote, respectively, the Fréchet derivatives of $\boldsymbol{L}_{\boldsymbol{d}}^{\mathrm{e}}, \boldsymbol{L}_{\boldsymbol{\theta}}^{\mathrm{e}}$ at $(\boldsymbol{d}, \boldsymbol{\theta})$. The above expressions are considered in the numerical experiments of Section 4. It should be noted, however, that the numerical methods proposed in this paper do not depend on a specific structure of the thin-walled solid operator and, hence, more realistic viscoelastic models can be considered (see, e.g., [45]).

Owing to the discussion of Section 2.1, the proposed time semi-discrete approximations of (28)-(29) are reported in Algorithm 2. The fluid domain geometry update is treated explicitly in 
the first step while the three subsequent steps perform a fully decoupled sequential computation of $\boldsymbol{u}^{n}, p^{n}$ and $\left(\boldsymbol{d}^{n}, \boldsymbol{\theta}^{n}\right)$.

Remark 11 Note that, by definition, all the time semi-discrete fluid fields $\left\{\tilde{\boldsymbol{u}}^{n}\right\}_{n \geq 0},\left\{\boldsymbol{u}^{n}\right\}_{n \geq 1}$, $\left\{\phi^{n}\right\}_{n \geq 0},\left\{p^{n}\right\}_{n \geq 0}$ and $\{\boldsymbol{w}\}_{n \geq 1}$ are defined in the reference configuration $\Omega$. In (30)-(31) these fields are transported to $\Omega^{n}$ by composition with $\left(\mathcal{A}^{n}\right)^{-1}$.

\section{Numerical experiments}

In this section we investigate the performance of the proposed fully decoupled schemes in several numerical examples. Section 4.1 focuses on the linear model problem (1)-(2) with a simple two-dimensional geometry. The nonlinear problem (28)-(29) and more complex geometries are considered in Sections 4.2-4.5.

The numerical tests of Section 4.1 are carried out using a computer implementation of Algorithm 1 based on FreeFem ++ (see [43]). The results of Sections 4.2-4.5 are obtained with a parallel implementation of Algorithm 2. A master/slave paradigm is used in which the master controls the data exchanges across the interface and the slaves are the fluid and structure parallel solvers. Different parallel approaches are used for the solid and the fluid. The parallel structural solver uses the Newton method at each time-step. The resulting linear systems are solved with a balancing domain decomposition method (see, e.g., [54, 50, 49]). The key point in this algorithm is the construction of the coarse space which, for robustness, is based on the stiffness matrix. The fluid solver FELiScE (see [47]), based on PETSc (see [9, 10, 11]), uses an additive Schwarz method (see, e.g., [68]) with local ILU prenconditionners. We will use the acronym "fds" (fully decoupled scheme) to refer to Algorithms 1 and 2 in the labels of the figures.

Remark 12 It should be noted that the worst-case scenario is considered for the numerical solution of steps 2 and 3 in Algorithm 2, in the sense that no dedicated preconditioners are used for each of them. The computational cost reductions reported in Sections 4.2-4.5 below can hence be improved by considering more efficient solvers for the projection schemes in the fluid.

\subsection{Convergence study in a two-dimensional test-case}

The first example simulates the propagation of a pressure-wave within an elastic straight tube in two-dimensions. Basically, we couple the Stokes system (1) with a viscoelastic string model, i.e., in (2) we take

$$
\begin{aligned}
& \boldsymbol{d}=\left(\begin{array}{l}
0 \\
\eta
\end{array}\right), \quad \boldsymbol{\theta}=\mathbf{0}, \quad \boldsymbol{L}_{\boldsymbol{\theta}}^{\mathrm{e}}(\boldsymbol{d}, \boldsymbol{\theta})=\mathbf{0}, \quad \boldsymbol{L}_{\boldsymbol{\theta}}^{\mathrm{v}}(\dot{\boldsymbol{d}}, \dot{\boldsymbol{\theta}})=\mathbf{0}, \\
& \boldsymbol{L}_{\boldsymbol{d}}^{\mathrm{e}}(\boldsymbol{d}, \boldsymbol{\theta})=\left(\begin{array}{c}
0 \\
-\lambda_{1} \partial_{x x} \eta+\lambda_{0} \eta
\end{array}\right), \quad \boldsymbol{L}_{\boldsymbol{d}}^{\mathrm{v}}(\dot{\boldsymbol{d}}, \dot{\boldsymbol{\theta}})=\left(\begin{array}{c}
0 \\
\alpha_{0} \rho^{\mathrm{s}} \epsilon \dot{\eta}-\alpha_{1} \lambda_{1} \partial_{x x} \dot{\eta}
\end{array}\right),
\end{aligned}
$$

with $\lambda_{1} \stackrel{\text { def }}{=} \frac{E \epsilon}{2(1+\nu)}$ and $\lambda_{0} \stackrel{\text { def }}{=} \frac{E \epsilon}{R^{2}\left(1-\nu^{2}\right)}$. Here, $R$ is the radius of the tube an $E, \nu$ respectively denote the Young modulus and the Poisson ratio of the solid. Note that, in this simplified setting, the tangential contributions involved in $(8)_{3}$ (or $\left.(7)_{1}\right)$ vanish (see also [33]).

For the fluid we take $\rho^{\mathrm{f}}=1.0$ and $\mu=0.035$, and for the solid $\rho^{\mathrm{s}}=1.1, \epsilon=0.1, E=$ $0.75 \cdot 10^{6}, \nu=0.5, \alpha_{0}=1$ and $\alpha_{1}=10^{-3}$. All units are expressed in the CGS system. The fluid domain is given by $\Omega=[0, L] \times[0, R]$ and the fluid-solid interface by $\Sigma=[0, L] \times\{R\}$, with $L=6$ and $R=0.5$. At $t=0$, the whole system is at rest. A sinusoidal pressure-wave (with maximum $2 \cdot 10^{4}$ ) is prescribed on the inlet boundary $x=0$ during $5 \cdot 10^{-3}$ time instants. Zero pressure is 

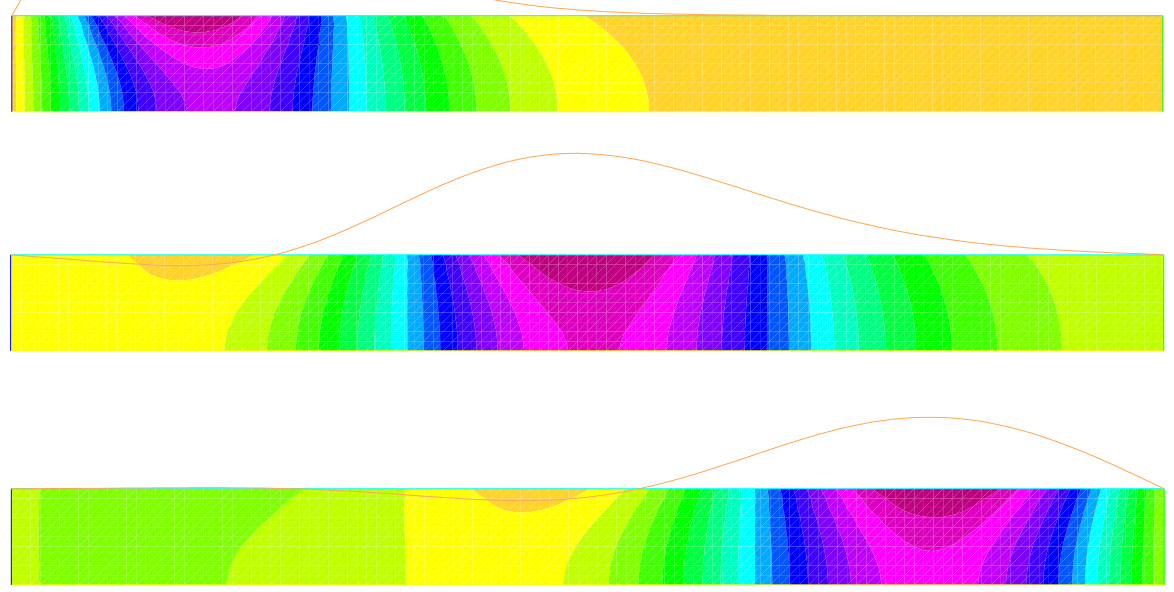

Figure 2: Snapshots of the fluid pressure and (exaggerated) solid displacement at time instants $t=0.005,0.01,0.015$ (from top to bottom). Algorithm 1 with $s=0, r=1, \tau=10^{-4}$ and $h=0.05$.

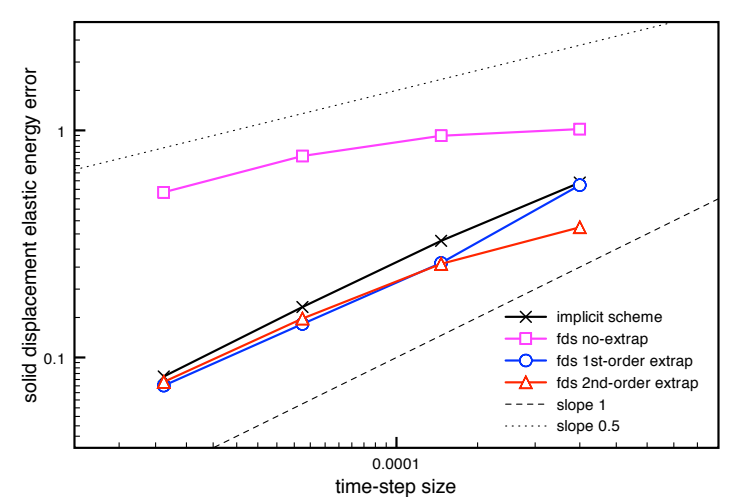

(a) $s=0$

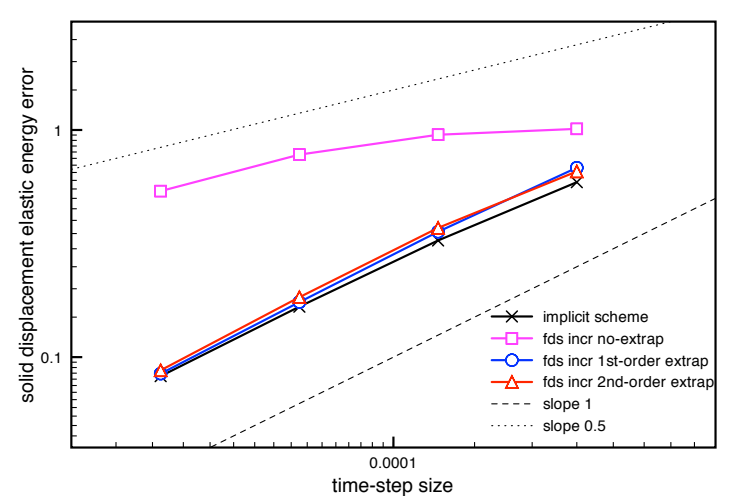

(b) $s=1$.

Figure 3: Time-convergence history of the displacement at $t=0.015$, with $h=\mathcal{O}(\tau)$, using the the non-incremental (a) and incremental (b) pressure-correction variants in Algorithm 1, and the implicit-scheme.

imposed at $x=L$ and a slip condition is enforced on the lower boundary $y=0$. For the solid we set $\eta=0$ at $x=0, L$. The spatial discretization of the fluid and of the structure is based on piece-wise affine continuous finite elements. In the case $s=1$, a Brezzi-Pitkäranta pressure stabilization (see, e.g., [15]) is added to step (9) of Algorithm 1.

Figure 2 presents the snapshots of the pressure field and the solid displacement (amplified by a factor 5 ) at the time instants $t=0.005,0.01$ and 0.015 , obtained from Algorithm 1 with $s=0, r=1, \tau=10^{-4}$ and $h=0.05$. The scheme is able to reproduce a stable pressure-wave propagation. Similar results, not reported here, are obtained with $s=1$. 


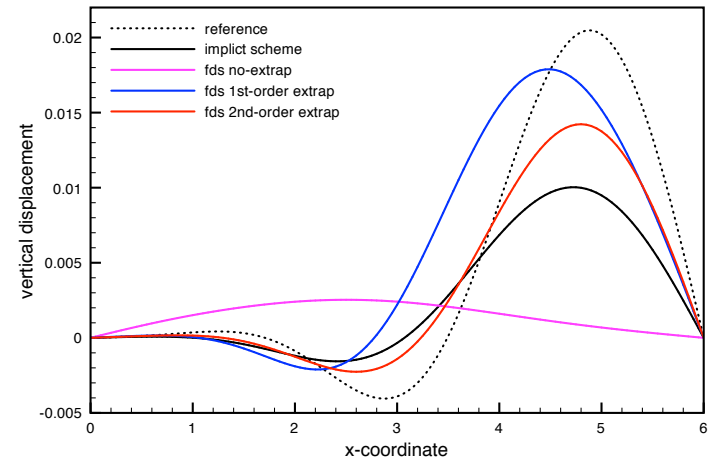

(a) $i=1$.

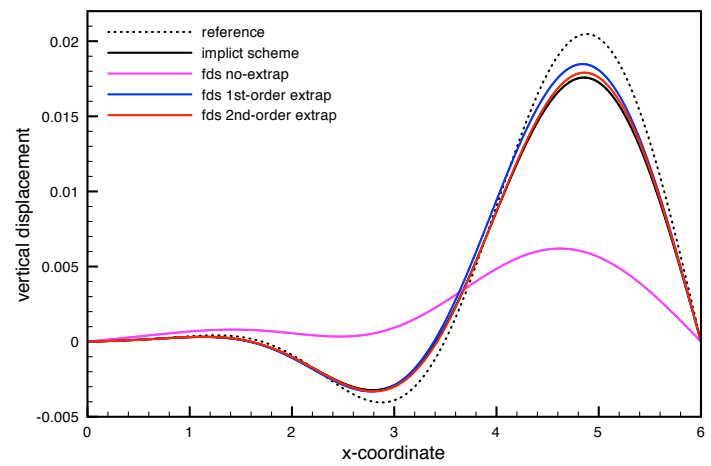

(c) $i=3$.

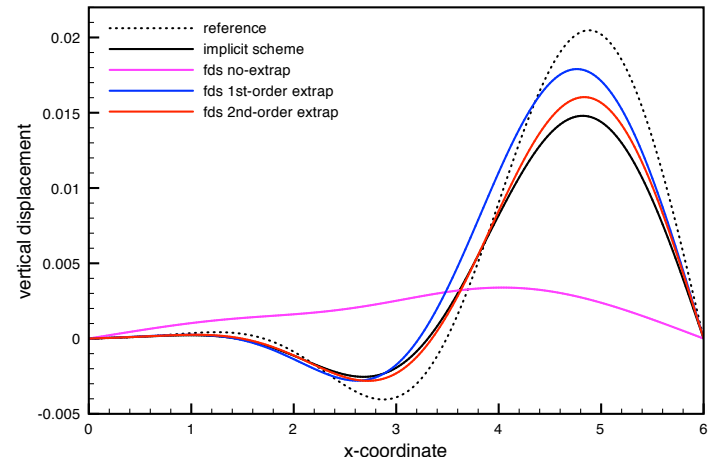

(b) $i=2$.

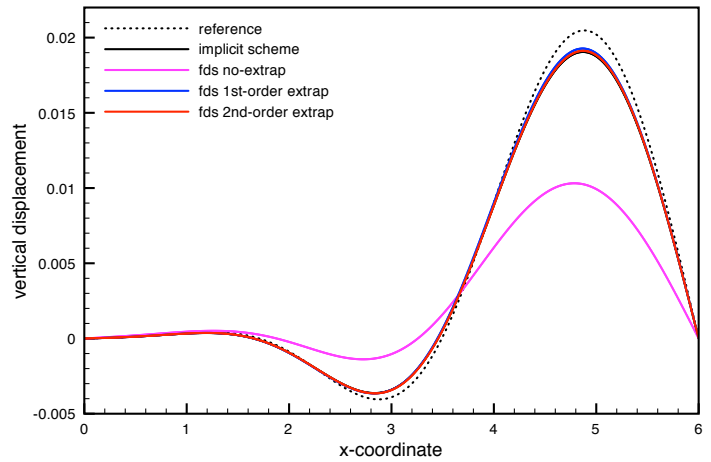

(d) $i=4$.

Figure 4: Comparison of the solid displacements at $t=0.015$ for different levels of $(\tau, h)$ refinement, given by (32), using Algorithm 1 with $s=0$ and the implicit-scheme.

In order to assess the overall convergence rate of Algorithm 1, we have uniformly refined in time and in space under a hyperbolic-CFL condition $(\tau=\mathcal{O}(h))$ :

$$
(\tau, h)=\left\{5 \cdot 10^{-4} / 2^{i}, 10^{-1} / 2^{i}\right\}_{i=1}^{4} .
$$

Figure 3 shows the relative elastic energy-norm error of the solid displacement, at time $t=0.015$, obtained with all the different variants of Algorithm 1. For comparison purposes the errors obtained with a fully implicit first-order scheme (including a monolithic time-stepping in the fluid) are also displayed. The reference solution has been computed with the implicit scheme and high space-time resolution: $h=3.125 \cdot 10^{-3}$ and $\tau=10^{-6}$. The results of Figure 3 indicate that the proposed fully decoupled schemes with $r=1$ or $r=2$ are able to retrieve the optimal first-order convergence rate $\mathcal{O}(\tau)$ of the implicit scheme, irrespectively of the choice $s=0$ or $s=1$ in the fluid time-steppping. Conversely, a sub-optimal overall rate $\mathcal{O}\left(\tau^{\frac{1}{2}}\right)$ is observed for $r=0$. We can also notice that the type of projection scheme in the fluid (i.e., $s=0,1$ ) has a limited impact on the overall accuracy of Algorithm 1, which is mainly driven by the choices of $r$ (see Remark 7). Further numerical evidence on these observations is given in Figures 4 and 5, which show the displacements at $t=0.015$ obtained with the implicit scheme and all the variants of Algorithm 1, for different levels of space-time refinement.

A similar behavior in terms of $r$ was observed in [36] with the original explicit Robin-Neumann 


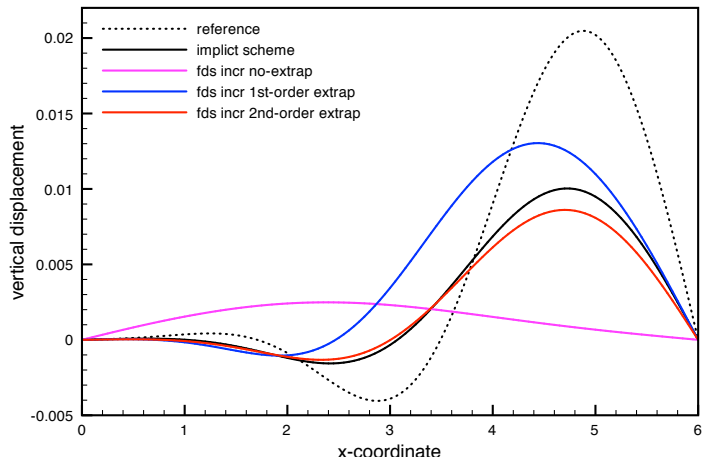

(a) $i=1$

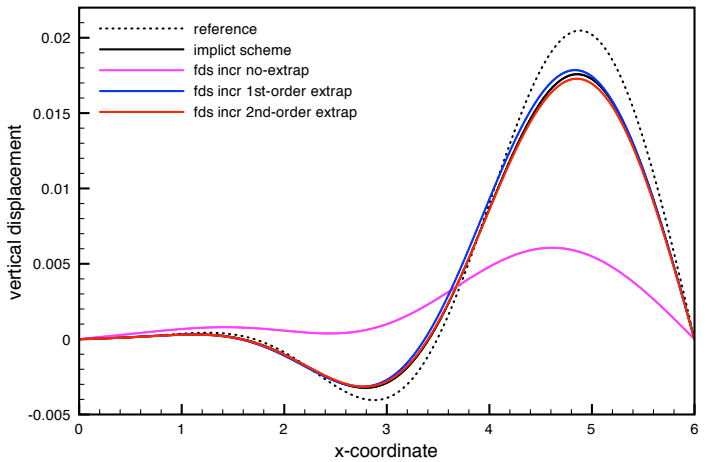

(c) $i=3$.

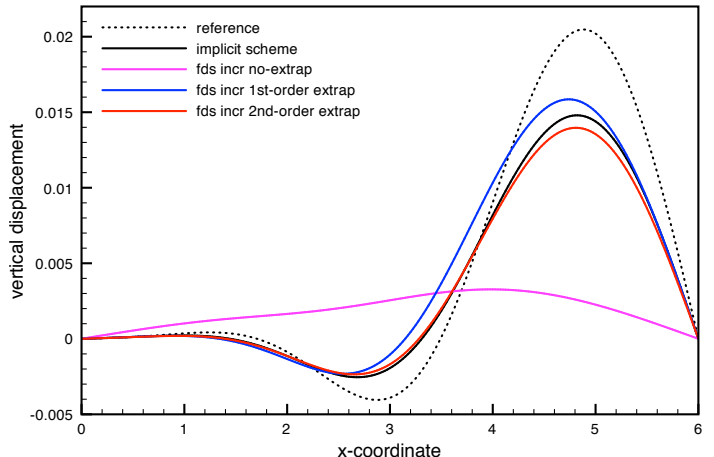

(b) $i=2$.

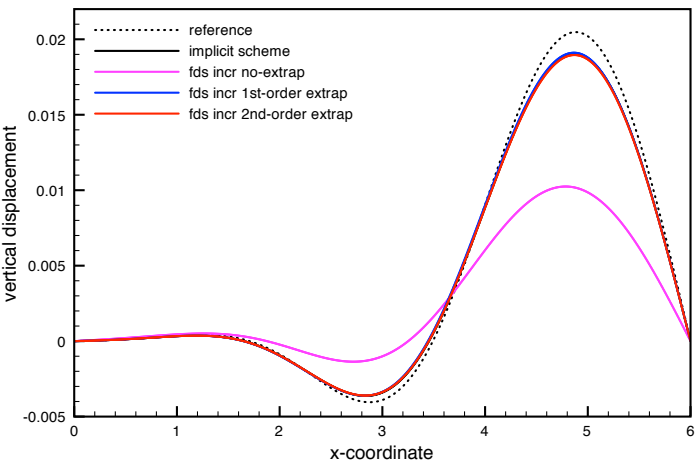

(d) $i=4$.

Figure 5: Comparison of the solid displacements at $t=0.015$ for different levels of $(\tau, h)$ refinement, given by (32), using Algorithm 1 with $s=1$ and the implicit-scheme.

schemes, hence indicating that the fully decoupled schemes proposed in the present work preserve their accuracy properties.

Remark 13 For the sake of conciseness, and owing to the above discussion, only the results obtained from Algorithm 2 with $s=0$ will be reported in the next sections.

\subsection{Pressure wave propagation in a straight tube}

The second numerical test is basically a three-dimensional non-linear version of the previous example (see also, e.g., [37]). The physical system is described by the non-linear coupled problem (28)-(29), with a Saint Venant-Kirchhoff constitutive law for the solid.

The fluid domain is a cylinder of radius $R=0.5$ and length $L=5$. All the units are expressed in the CGS system. The physical parameters of the fluid are $\rho^{\mathrm{f}}=1.0$ and $\mu=0.035$. For the structure we take $\rho^{\mathrm{s}}=1.2, \epsilon=0.1, E=3 \cdot 10^{6}$ and $\nu=0.5$. Damping effects in the solid are neglected in this case. A constant pressure of $1.3332 \cdot 10^{4}$ is imposed on the inlet boundary during $5 \cdot 10^{-3}$ time instants, thereafter this values is set to zero. An homogeneous natural boundary condition is prescribed on the outlet boundary. The whole fluid-solid system is initially at rest. Both the velocity and the pressure are discretized in space using $\mathbb{Q}_{1}$ finite elements. A streamline-upwind/Petrov-Galerkin (SUPG) stabilization is applied to the viscous 

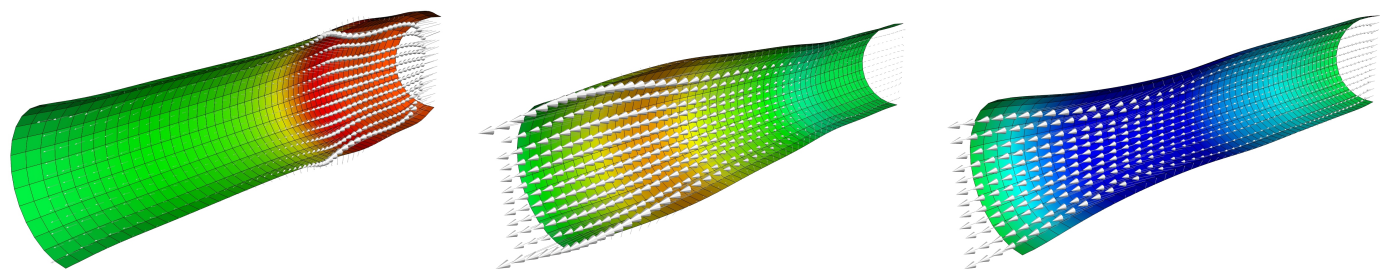

Figure 6: Snapshots of the velocity field and fluid interface pressure at $t=0.005,0.01,0.015$ (from left to right). Algorithm 2 with $s=0, r=1, \tau=10^{-4}$ and $h \approx 0.1$.

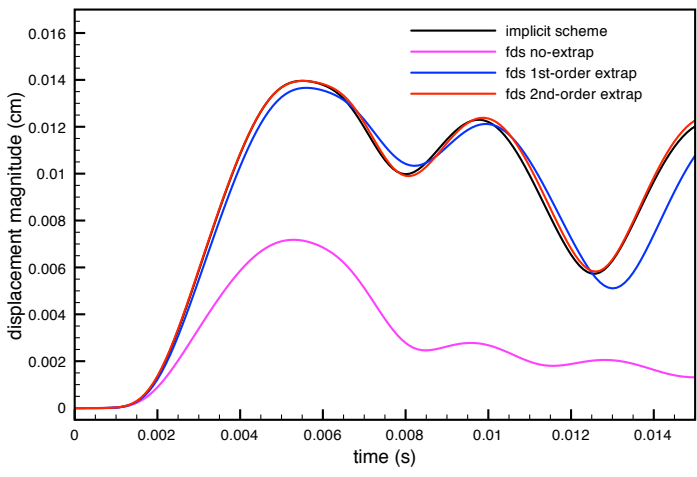

(a) $\tau=10^{-4}, h \approx 0.1$.

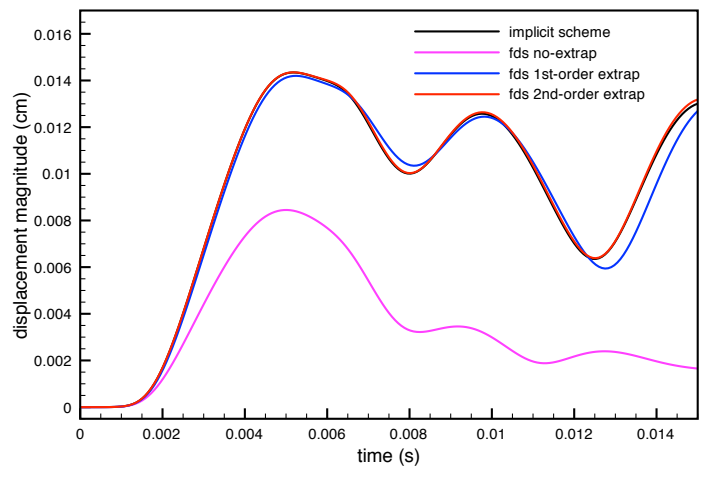

(b) $\tau=7.5 \cdot 10^{-5}, h \approx 0.07$.

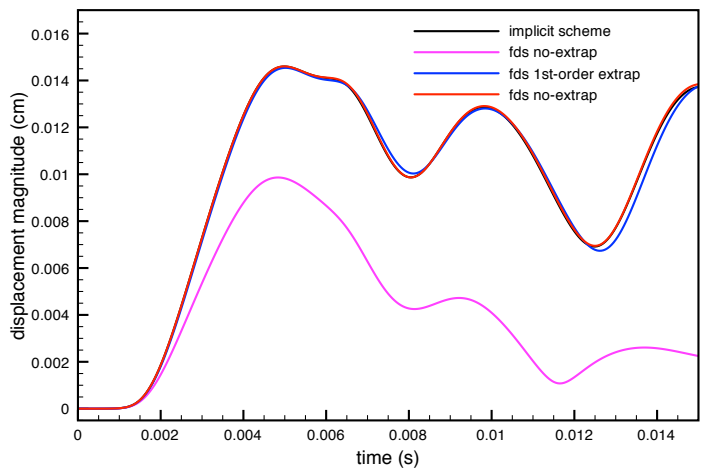

(c) $\tau=5 \cdot 10^{-5}, h \approx 0.05$

Figure 7: Comparison of the solid displacements at $t=0.015$ for different levels of space-time refinement. Algorithm 2 with $s=0$ and the implicit coupling scheme.

sub-step (30) of Algorithm 2. The shell equation is discretized in space by quadrilateral MITC4 elements (see [21, Section 8.2.1]).

We first consider Algorithm 2 with $s=0, r=1, \tau=10^{-4}$ and $h \approx 0.1$. Figure 6 shows the fluid velocity field and the solid deformation (amplified by a factor 10) retrieved at the time instants $t=0.005,0.01$ and 0.015. A stable propagating pressure-wave is obtained.

We now turn the discussion back to the accuracy of the methods. For this purpose, we have reported in Figure 7 the time history of the midpoint displacements obtained from Algorithm 2 with $s=0$ and a fully implicit first-order scheme for different levels of space-time refinement, 
namely: $\tau=10^{-4}, h \approx 0.1 ; \tau=7.5 \cdot 10^{-5}, h \approx 0.07$; and $\tau=5 \cdot 10^{-5}, h \approx 0.05$. The convergent behavior of the two extrapolated explicit variants (i.e., $r=1$ and $r=2$ ) towards the implicit coupling solution is clearly noticeable. On the contrary, the scheme with $r=0$ delivers a much slower convergent behavior. This confirms the convergence rates observed in Section 4.1.

\begin{tabular}{|c|c|c|}
\cline { 2 - 3 } \multicolumn{1}{c|}{} & Algorithm 2 & Implicit scheme \\
\hline$\tau=10^{-4}, h \approx 0.1$ & 1 & 10.5 \\
\hline$\tau=7.5 \cdot 10^{-5}, h \approx 0.07$ & 3.8 & 31.1 \\
\hline$\tau=5 \cdot 10^{-5}, h \approx 0.05$ & 12.4 & 80.6 \\
\hline
\end{tabular}

Table 1: Elapsed CPU-time (dimensionless) for different space-time refinements.

In Table 1 we have reported the elapsed CPU-times (dimensionless) obtained with Algorithm 2 $(s=0, r=1)$ and the implicit scheme. For the latter, the resulting coupled non-linear system at each time-step is solved in a partitioned fashion using a Robin-Neumann iterative procedure (see [4, 36]). We can observe that, for a similar level of accuracy, Algorithm $2(s=0, r=1)$ is about 8 times faster than the implicit solver in this case.

\subsection{Damped structural instability with a fully enclosed fluid}

We consider the example proposed in [48] where an incompressible fluid is confined within two curved thin-walled structures of different stiffness. The physical system is described by the non-linear coupled problem (28)-(29), with a Saint Venant-Kirchhoff constitutive relation for the (undamped) shell. We take $\rho^{\mathrm{f}}=1.0$ and $\mu=9$ in the fluid problem and $\rho^{\mathrm{s}}=500, \epsilon=$ $0.1, E_{\text {top }}=9 \cdot 10^{5}, E_{\text {bottom }}=9 \cdot 10^{8}$ and $\nu=0.3$. in the solid (the subscript notation indicates the bottom and top structures). The units are expressed in the SI system. On the left and right inflow boundaries, constant parabolic velocity profiles with maximal magnitudes 10 and 10.1 are, respectively, imposed. Homogeneous Dirichlet conditions are enforced on the remaining fluid boundaries. A vertical force of unitary magnitude loads downwards the fluid. We consider Algorithm 2 with the same spatial discretization as in the previous example. The simulations are carried out in three-dimensions by prescribing symmetry conditions on the extruded boundaries.

Figure 8 reports the fluid velocity magnitude at the time instants $t=0.5,1,1.5,2,2.5,3,3.5$ and 4 , using Algorithm 2 with $s=0, r=1, \tau=0.005$ and $h \approx 0.07$. We can clearly observe that the excess of fluid mass causes first the deflection of the upper (less stiff) structure. The fluid cavity continues to swell along the upper boundary until the fluid pressure reaches a critical level at which the (stiffer) lower structure is no longer able to resist and collapses. A similar behavior was observed in [48], using an implicit coupling solution method based on partitioned DirichletNeumann iterations and an augmented procedure, which prescribes a volume constraint on the structural system. In Algorithm 2, the Robin condition (31) 3 removes the indetermination of the fully enclosed fluid pressure.

For comparison purposes, we have reported in Figure 9 the interface mid-point displacement magnitude of the lower structure obtained with Algorithm $2(s=0, r=0,1,2)$ and a first-order implicitly coupled scheme (including a monolithic time-stepping in the fluid), for the following values of $\tau$ and $h: \tau=10^{-2}, h \approx 0.2 ; \tau=5 \cdot 10^{-3}, h \approx 0.07$ and $\tau=2.5 \cdot 10^{-3}, h \approx 0.04$. The lack of accuracy for the scheme with $r=0$ is striking. Even after two space-time refinements, the unphysical excess of mass-loss across the interface impedes the collapse of the bottom structure. Conversely, the fully decoupled schemes with $r=1$ and $r=2$ are able to retrieve the accuracy of the implicit scheme.

In order to illustrate the efficiency of the proposed methods, the elapsed CPU-times, for the above degrees of space and time refinement, are provided in Table 2. We compare Algorithm 2 

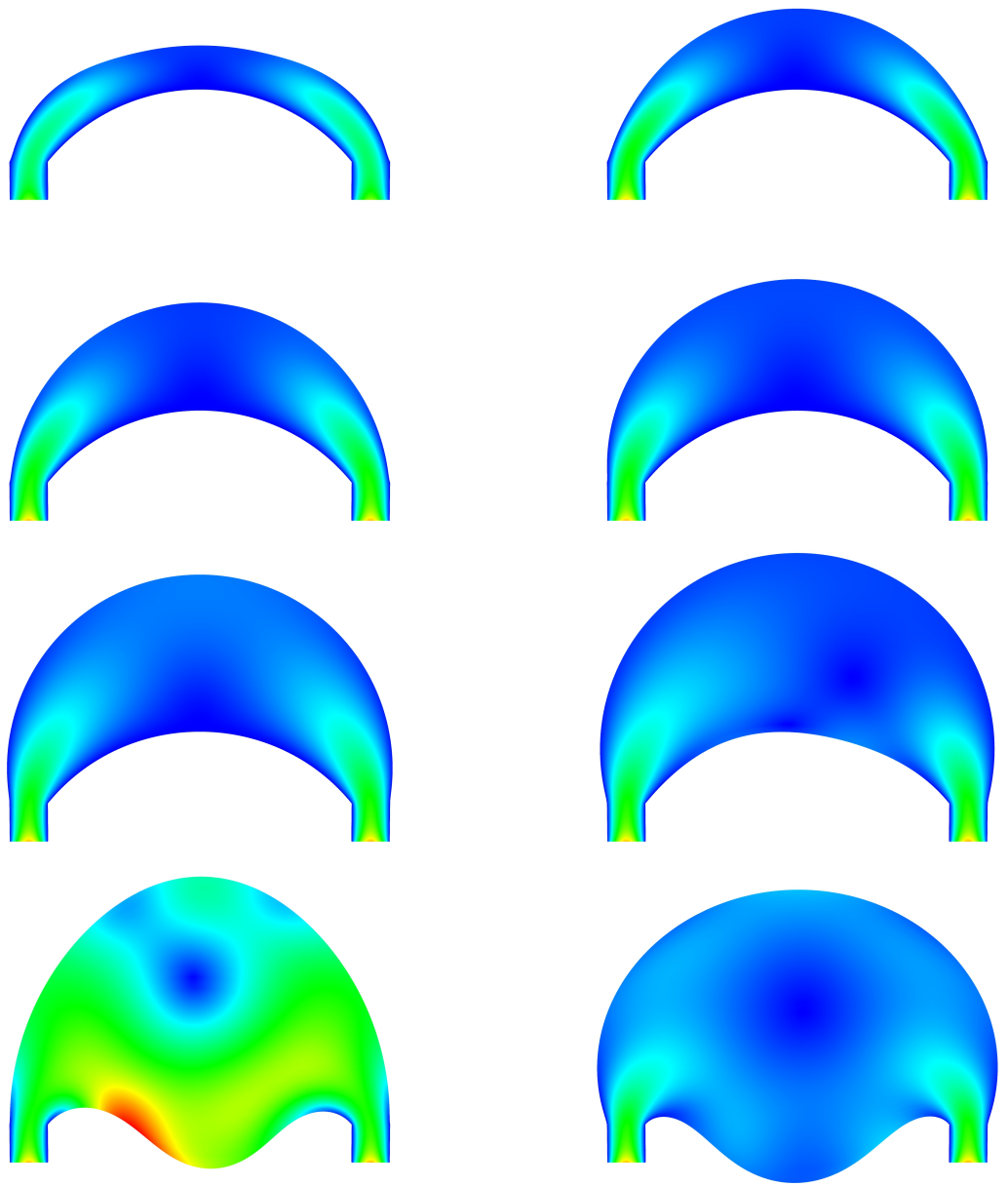

Figure 8: Snapshots of the fluid velocity at the time instants $t=0.5,1,1.5,2,2.5,3,3.5,4$ (from left to right and top to bottom). Algorithm 2 with $s=0, r=1, \tau=0.005$ and $h \approx 0.07$.

\begin{tabular}{|c|c|c|}
\cline { 2 - 3 } \multicolumn{1}{c|}{} & Algorithm 2 & Implicit scheme \\
\hline$\tau=10^{-2}, h \approx 0.2$ & 1 & 12.5 \\
\hline$\tau=5 \cdot 10^{-3}, h \approx 0.07$ & 13.5 & 162 \\
\hline$\tau=2.5 \cdot 10^{-3}, h \approx 0.04$ & 92.5 & 1200 \\
\hline
\end{tabular}

Table 2: Elapsed CPU-time (dimensionless)

$(s=0, r=1)$ and the implicit scheme solved, as in the previous example, with a partitioned Robin-Neumann procedure. For a similar level of accuracy (see Figure 9(b)), the speed-up provided by the fully decoupled scheme is around 12 times faster than the implicit solver. 


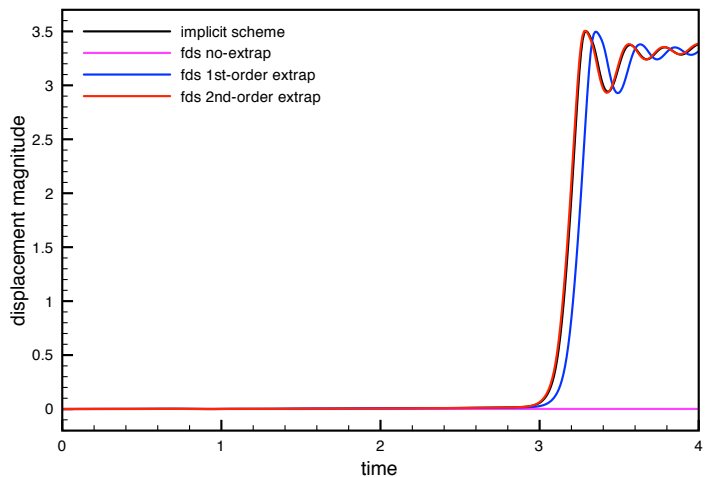

(a) $\tau=10^{-2}, h \approx 0.2$.

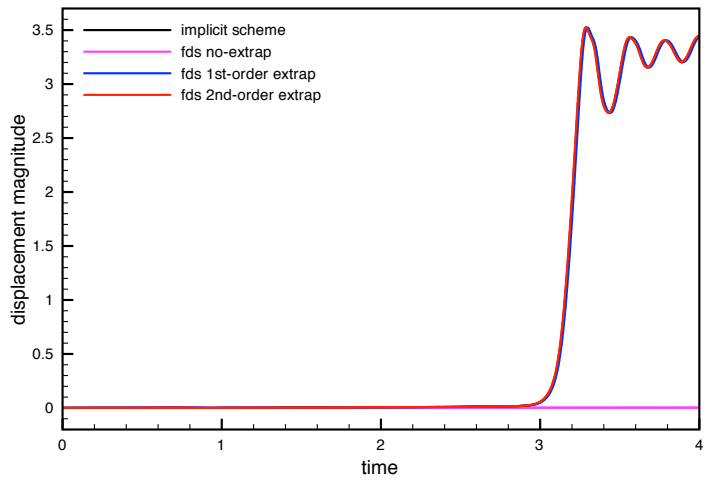

(b) $\tau=5 \cdot 10^{-3}, h \approx 0.07$.

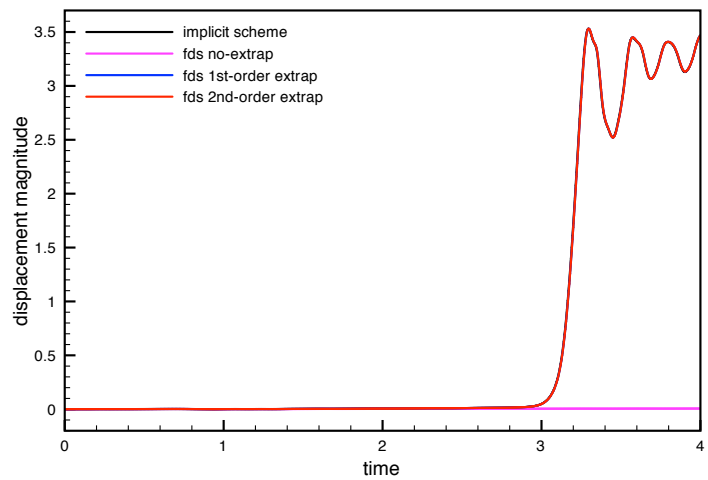

(c) $\tau=2.5 \cdot 10^{-3}, h \approx 0.04$.

Figure 9: Comparison of the bottom interface mid-point displacement vs. time for different levels of space-time refinement. Algorithm 2 with $s=0$ and the implicit coupling scheme.

\subsection{Blood flow in an abdominal aortic aneurysm}

In this example we simulate the blood flow within an in-vitro abdominal aortic aneurysm geometry (see, e.g., [67, 2, 36]). The physical system is here described by the coupled problem (28)-(29),
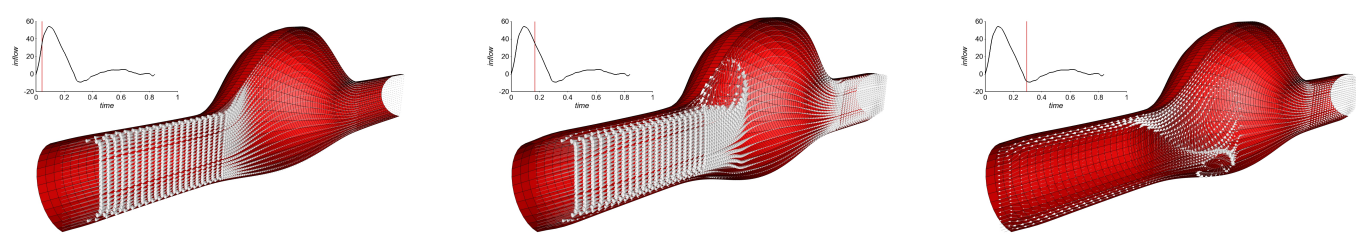

Figure 10: Snapshots of the fluid velocity field at the time instants $t=0.042,0.168,0.294$ (from left to right). Algorithm 2 with $s=0, r=1, \tau=4.2 \cdot 10^{-4}$ and $h \approx 0.2$.

with a Saint Venant-Kirchhoff constitutive relation for the aneurysm wall. The fluid-solid interface has a length of 22.95 and a uniform thickness of $\epsilon=0.17$. All units are expressed in the CGS system. We take $\rho^{\mathrm{f}}=1.0$ and $\mu=0.035$ in the fluid and $\rho^{\mathrm{s}}=1.2, E=3 \cdot 10^{6}, \nu=0.5, \alpha_{0}=1$ 


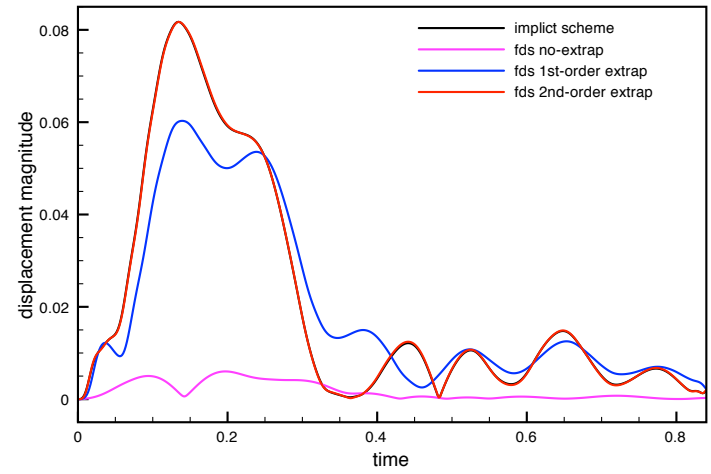

(a) $\tau=4.2 \cdot 10^{-4}, h \approx 0.2$.

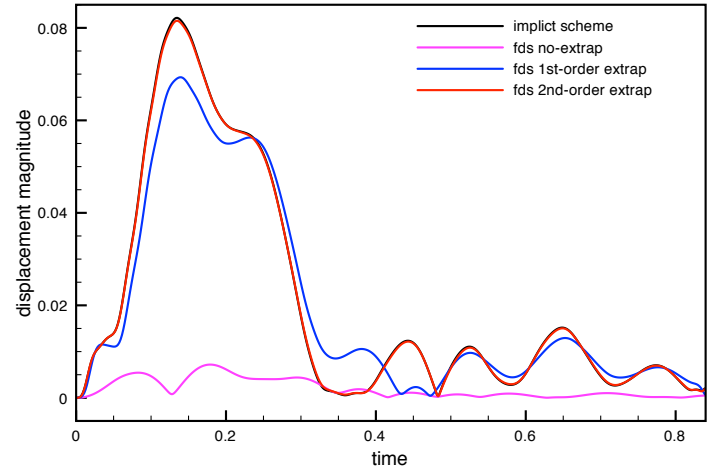

(b) $\tau=3 \cdot 10^{-4}, h \approx 0.15$.

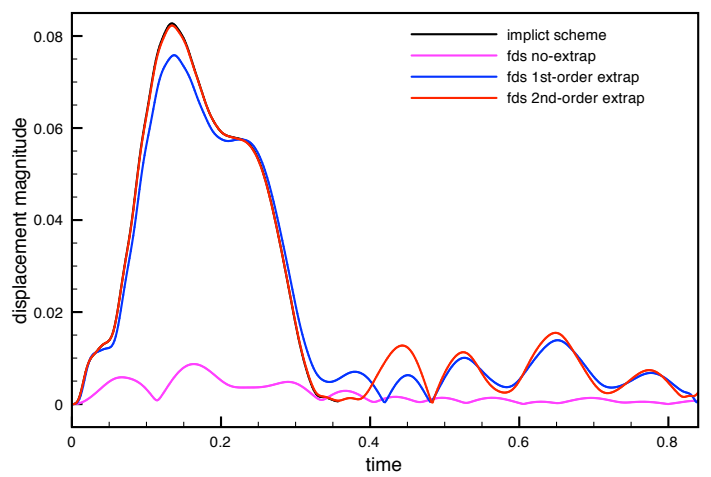

(c) $\tau=2.1 \cdot 10^{-4}, h \approx 0.1$.

Figure 11: Comparison of the solid displacements at $t=0.015$ for different levels of space-time refinement. Algorithm 2 with $s=0$ and the implicit coupling scheme.

and $\alpha_{1}=10^{-3}$ in the solid. The fluid and structure are initially at rest. On the inlet boundary, we impose a physiological flow rate waveform (see Figure 10) measured at the level of the infrarenal aorta (see [60]). A resistance boundary condition is enforced on the outlet boundary. The value of the resistance is set to $R_{\text {out }}=600$.

We consider Algorithm 2 with the same spatial discretization as in the previous example. The simulations are performed over a full cardiac cycle ( 0.84 seconds). For illustration purposes, the velocity field and the solid deformation at $t=0.042,0.168,0.294$, obtained from Algorithm 2 with $s=0, r=1, \tau=4.2 \cdot 10^{-4}$ and $h \approx 0.2$ are displayed in Figure 10 (half the geometry), showing a stable numerical approximation.

In Figure 11, the interface mid-point displacements are compared with those obtained with a first-order implicit coupling scheme, for different values of $\tau$ and $h$, namely: $\tau=4.2 \cdot 10^{-4}$, $h \approx 0.2 ; \tau=3 \cdot 10^{-4}, h \approx 0.15$ and $\tau=2.1 \cdot 10^{-4}, h \approx 0.1$. The low accuracy of the fully decoupled scheme with $r=0$ is, once more, striking: the numerical solution has an extremely poor accuracy, even with the finest discretization, which makes the scheme useless in practice. On the contrary, the convergent behavior of the variant with $r=1$ is clearly visible and delivers much more reasonable results (see Figure 11(b)). The fully decoupled scheme with $r=2$ gives practically the same accuracy than the implicit scheme.

At last, in Table 3, we compare the performance of the proposed fully decoupled scheme 


\begin{tabular}{|c|c|c|}
\cline { 2 - 3 } \multicolumn{1}{c|}{} & Algorithm 2 & Implicit scheme \\
\hline$\tau=4.2 \cdot 10^{-4}, h \approx 0.2$ & 1 & 41.8 \\
\hline$\tau=3 \cdot 10^{-4}, h \approx 0.15$ & 3.4 & 192 \\
\hline$\tau=2.1 \cdot 10^{-4}, h \approx 0.1$ & 10 & 671.7 \\
\hline
\end{tabular}

Table 3: Elapsed CPU time (dimensionless).

$(s=0, r=1)$ and the implicit scheme. Instead of the Robin-Neumann iterative procedure considered in the previous examples, here the implicit scheme is solved via a (parameter-free) Dirichlet-Neumann GMRES partitioned Newton method (see, e.g., [34]). The reason is that, in this case, the convergence speed of the Robin-Neumann iterations appeared to be highly sensitive to the value of the Robin coefficient and, hence, required careful tuning. As shown in Table 3, the savings in terms of computational effort are striking. For the finest discretizations, for which we obtain comparable levels of accuracy, the fully decoupled scheme is around 60 times faster than the implicit method.

\subsection{Blood flow in a patient-specific aorta}

The last numerical example is devoted to the numerical simulation of blood flow within a patientspecific thoracic aorta (with a mild coarctation) under physiological rest conditions. The computational 3D geometry (see Figure 12) and the inlet fluid flow rate are obtained from the data provided by the 2nd CFD Challenge organized within the STACOM 2013 conference (see [1]). In this example, the CGS system is adopted for all the physical units.

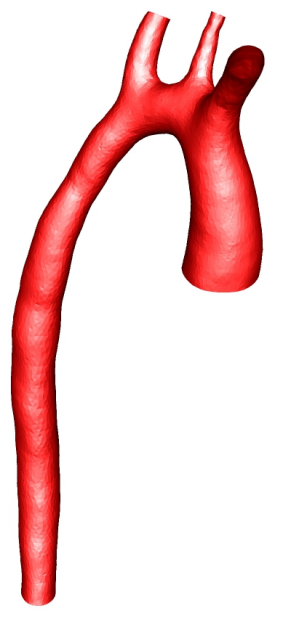

(a) Given stressed configuration

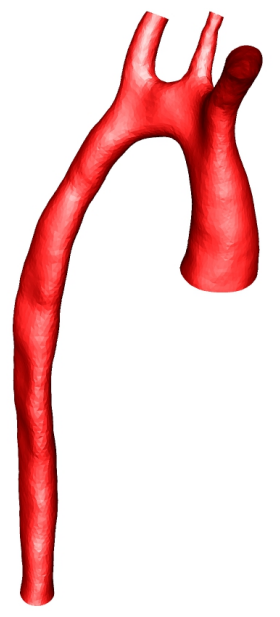

(b) Inferred stress-free configuration.

Figure 12: Reference configurations for the ALE map (a) and solid (b).

The mechanical interaction between blood and the aortic wall is modeled by the coupled system (28)-(29), where a Saint Venant-Kirchhoff model is used as constitutive relation for the aortic wall. Note that the latter model assumes a stress-free solid reference configuration $\Sigma$. The reconstructed aorta geometry, see Figure 12(a), corresponds to a deformed in vivo stress condition (obtained from magnetic resonance angiography). The ambient pressure for the rest state is 67000. In order to perform the simulation with physiological pressure levels, a stress-free solid 


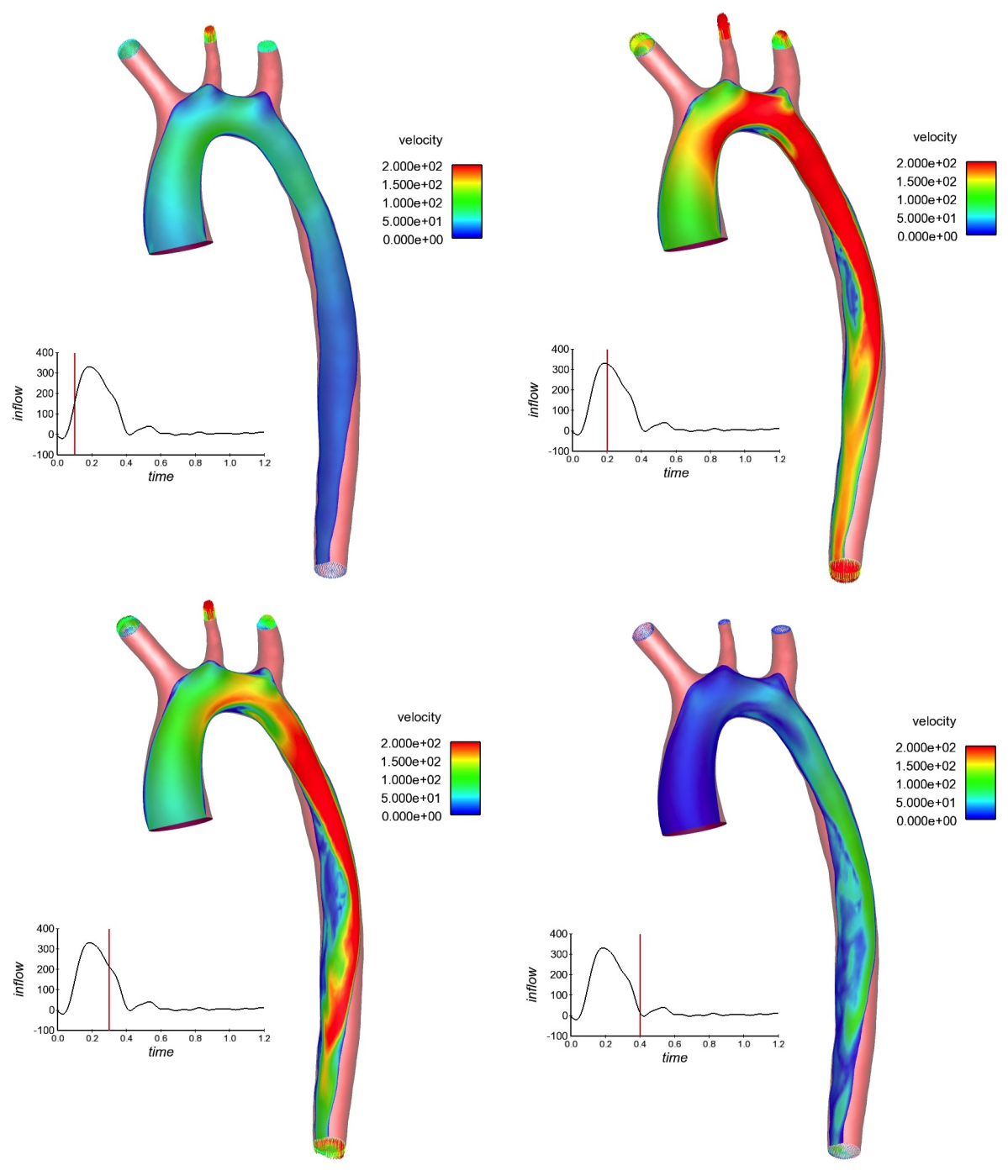

Figure 13: Snapshots of the fluid velocity at the time instants $t=0.1,0.2,0.3,0.4$ (from left to right and top to bottom). Algorithm 2 with $s=0$ and $r=1$.

reference configuration $\Sigma$ is inferred from the provided configuration (Figure 12(a)) by solving an inverse solid problem (see, e.g., [38, 55]), in such a way that the in vivo stressed configuration is retrieved when the rest ambient pressure conditions are prescribed on the unknown stress-free reference configuration (see Figure 12(b)). We refer to this reference displacement as $\boldsymbol{d}_{\boldsymbol{\sigma}_{0}}$.

The aortic wall is assumed to have uniform thickness and density of $\epsilon=0.2$ and $\rho^{\mathrm{s}}=1.2$, with the mechanical parameters set to $E=3 \cdot 10^{6}, \nu=0.5, \alpha_{0}=1$ and $\alpha_{1}=10^{-3}$. The blood dynamic viscosity and density are $\mu=0.04$ and $\rho^{\mathrm{f}}=1$. On the inlet boundary (ascending aorta), we prescribe the physiological fluid flow rate provided in [1] for the rest condition. On the remaining outlet boundaries (innominate, left carotid, left subclavian and descending aorta), explicit RCR Windkessel 0D-models are used to take into account the effect of the surrounding vascular network. The Windkessel parameters are those reported in [62]. The initial displacement 


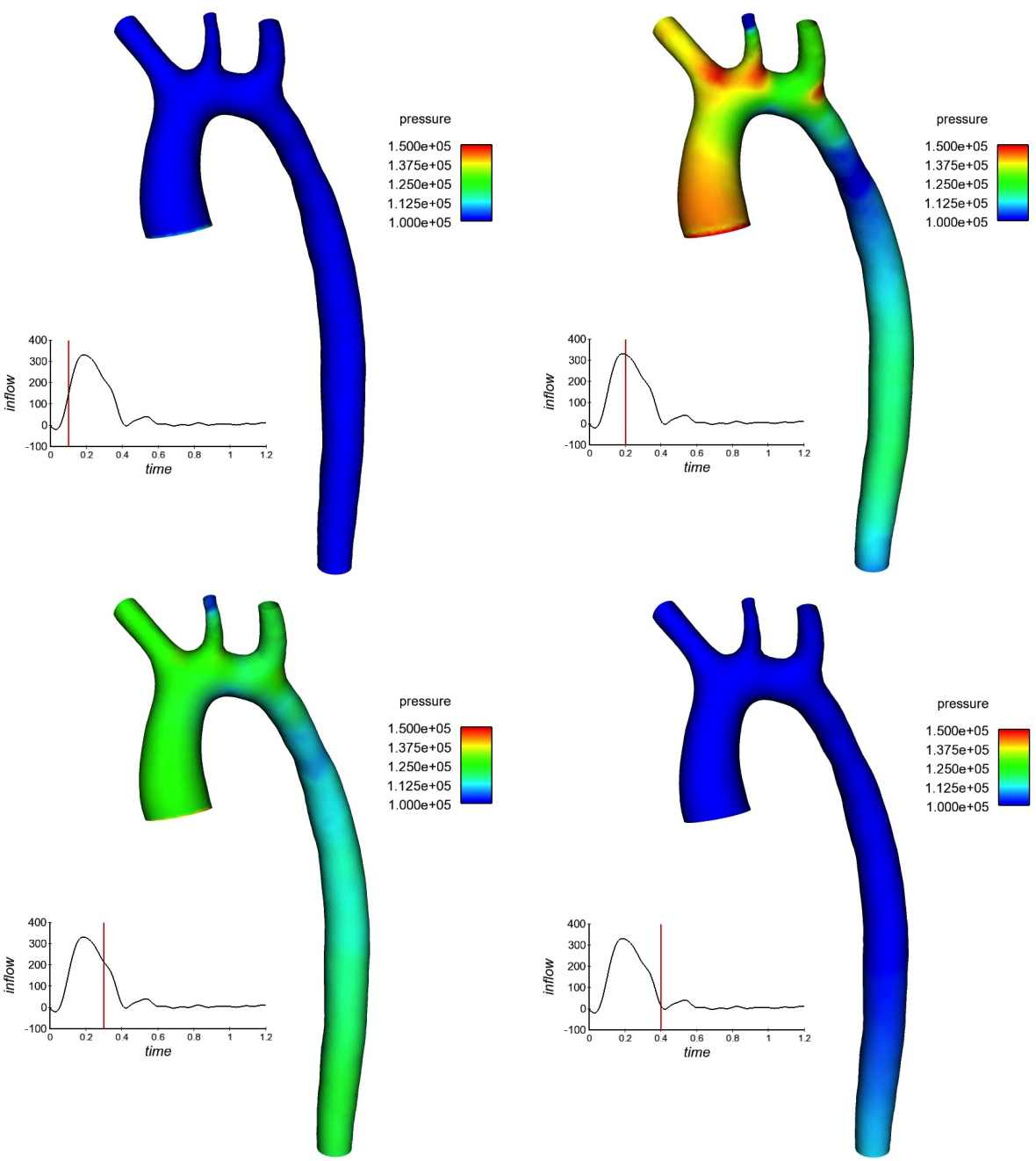

Figure 14: Snapshots of the fluid pressure at the time instants $t=0.1,0.2,0.3,0.4$ (from left to right and top to bottom). Algorithm 2 with $s=0$ and $r=1$.

of the structure equation (29) is set to $\boldsymbol{d}_{0}=\boldsymbol{d}_{\boldsymbol{\sigma}_{0}}$. The remaining fluid-structure system unknowns are initialized to zero.

All the fluid steps of Algorithm 2 are discretized in space using affine finite elements. Triangular MITC3 elements are used for the shell problem (see [21, Section 8.2.1]). The adapted fluid mesh (from [62]) is made of 375149 tetrahedra. The resulting matching solid mesh is made of 21752 triangles. We have simulated $1.2 \cdot 10^{4}$ time-steps of size $\tau=10^{-4}$, which corresponds to a full cardiac cycle. Figures 13 and 14 present, respectively, some snapshots of the fluid velocity and pressure fields obtained from Algorithm $2 s=0$ and $r=1$ at four different time instants of a cardiac cycle. A stable numerical solution is obtained which shows physiological values in both the velocity and pressure fields. To the best of our knowledge, this is the first time that such a blood flow simulation is performed with an explicit coupling scheme.

To illustrate the accuracy of the results, in Figures 15-17 we compare the maximum magnitude 

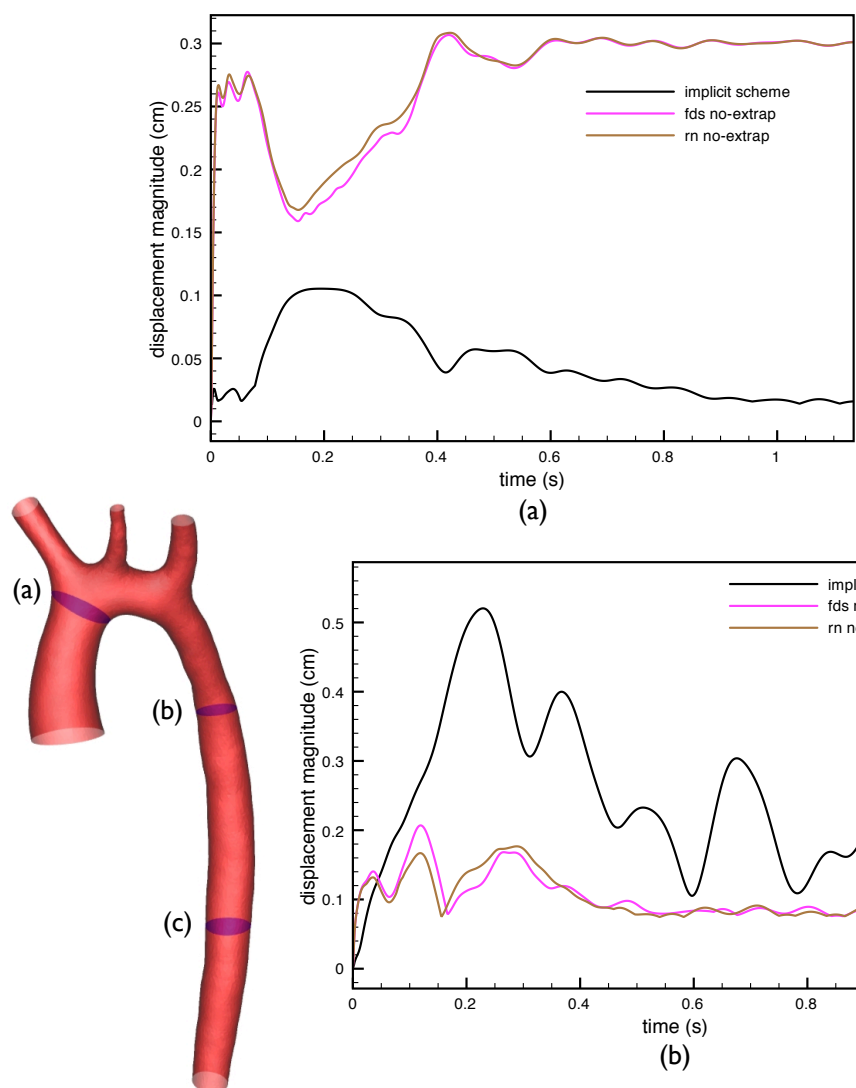

(a)

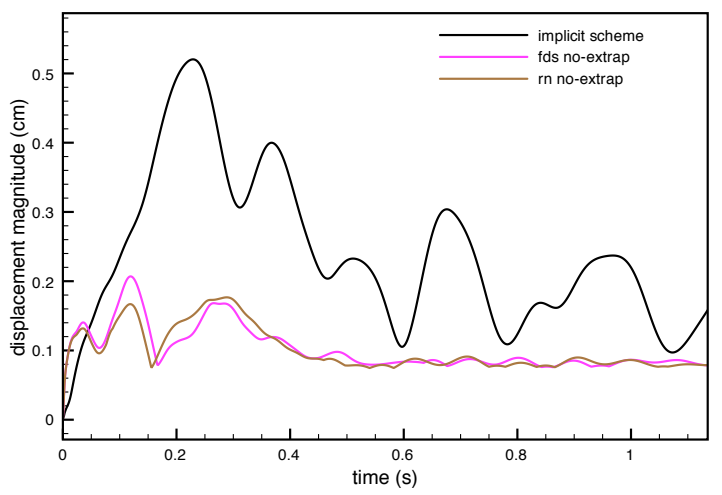

(b)

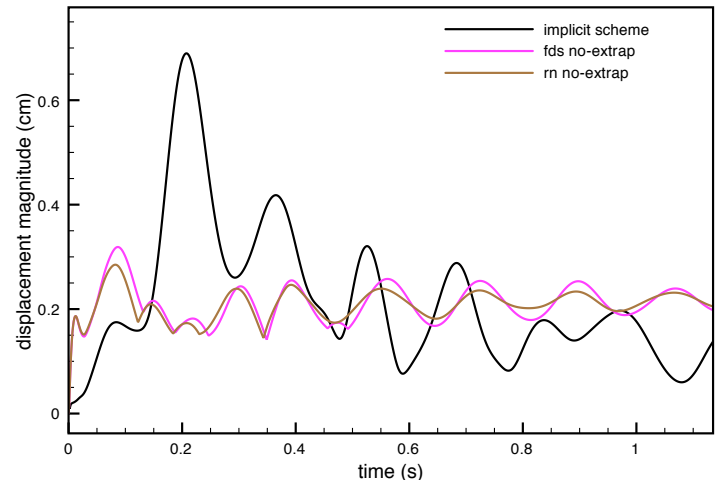

(c)

Figure 15: Maximum displacement magnitude at sections $(a),(b)$ and $(c)$ of the structure vs. time. Comparison of the implicit scheme, Robin-Neumann scheme (from [36, Algorithm 4]) with $r=0$, and Algorithm 2 with $s=r=0$.

of the displacement, at three different sections of the aorta, obtained with the implicit scheme, the original Robin-Neumann schemes (from [36, Algorithm 4]) and Algorithm 2 with $s=0$ and $r=0,1,2$, respectively. For comparison purposes, the results of the implicit scheme are reported in the three figures. We retrieve basically the same accuracy behavior than in the previous 

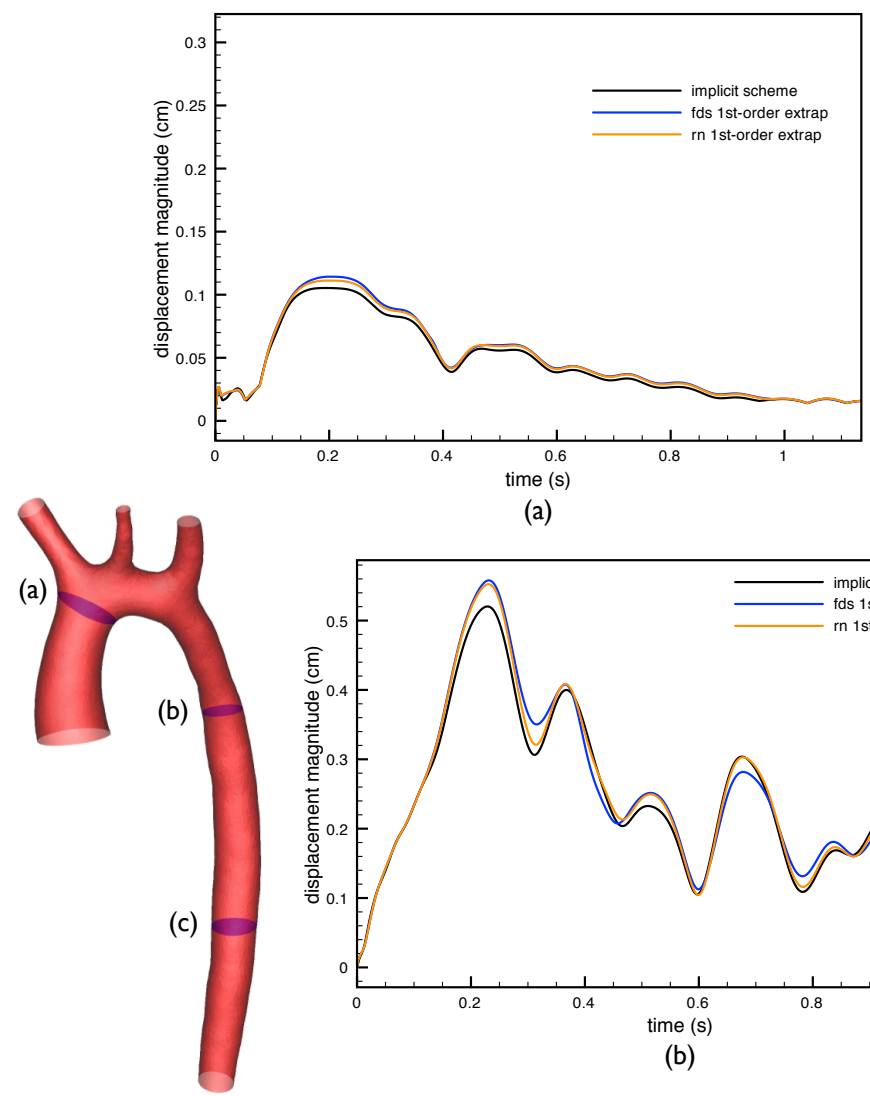

(a)

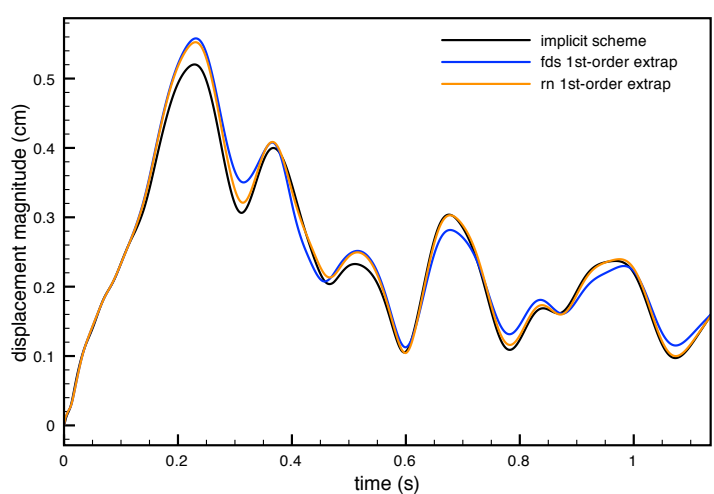

(b)

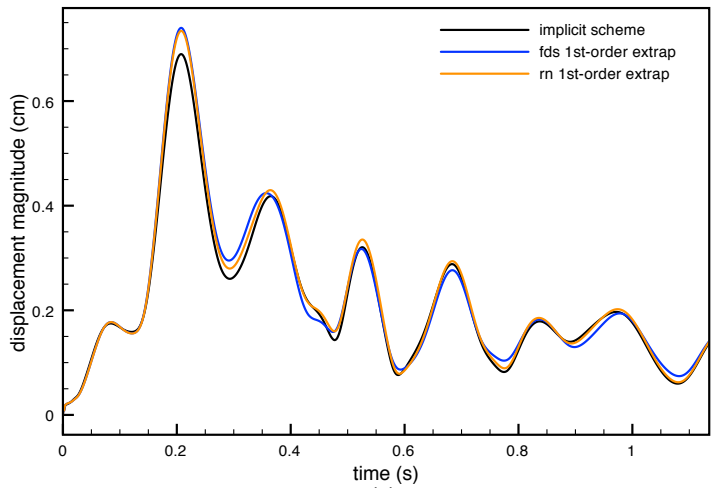

(c)

Figure 16: Maximum displacement magnitude at sections $(a),(b)$ and $(c)$ of the structure vs. time. Comparison of the implicit scheme, Robin-Neumann scheme (from [36, Algorithm 4]) with $r=1$, and Algorithm 2 with $s=0$ and $r=1$.

examples. Figure 15 shows that the Robin-Neumann schemes and Algorithm 2 with $s=r=0$ are unable to retrieve the overall dynamics of the implicit solution and yield approximations with extremely poor accuracy. The variants with interface extrapolations $r=1$ and $r=2$, whose results are shown in Figures 16 and 17, give practically the same accuracy as the implicit 

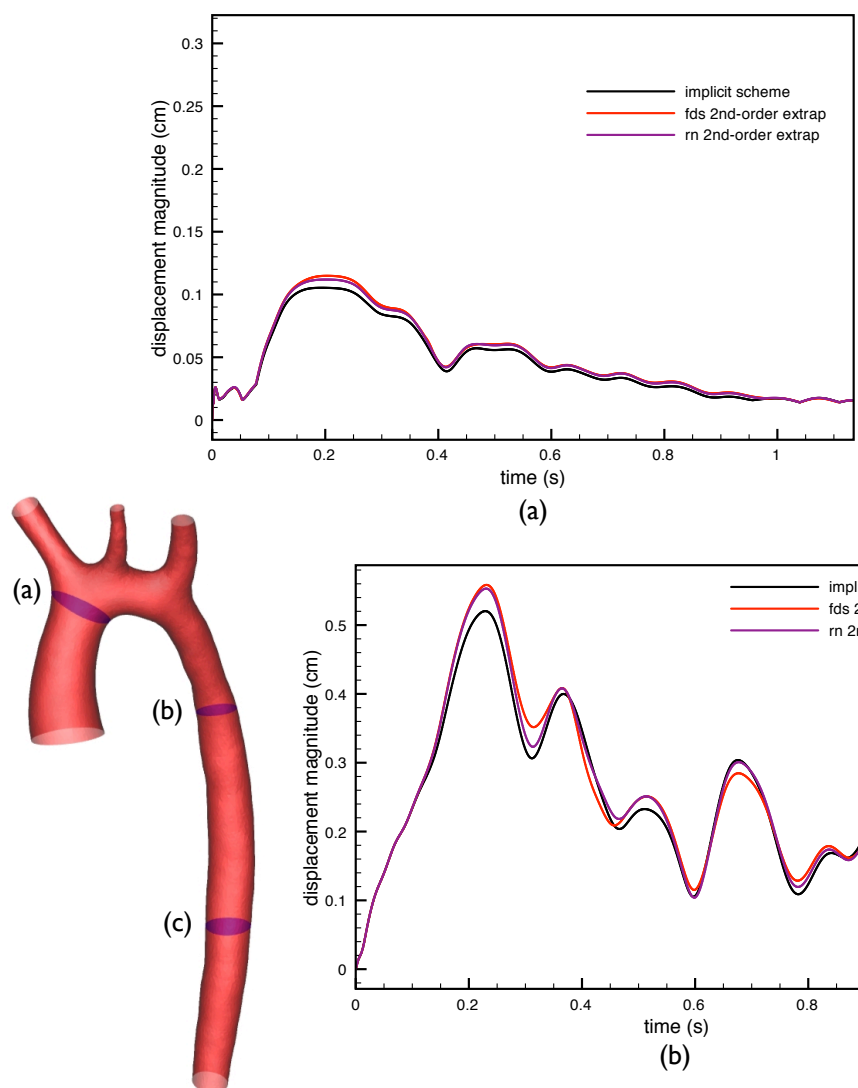

(a)

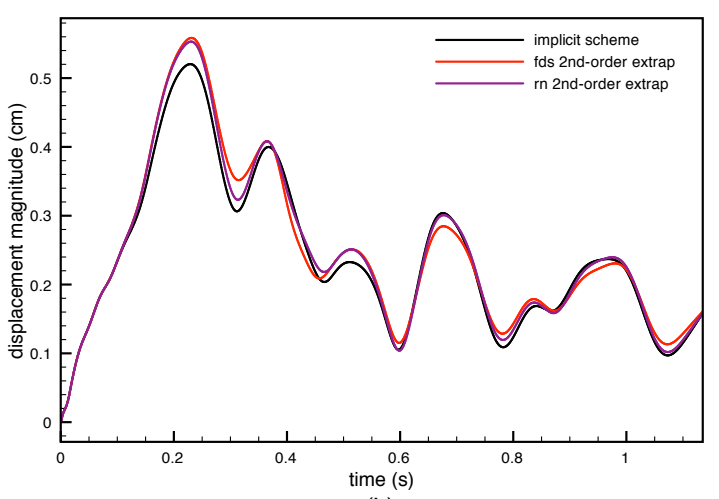

(b)

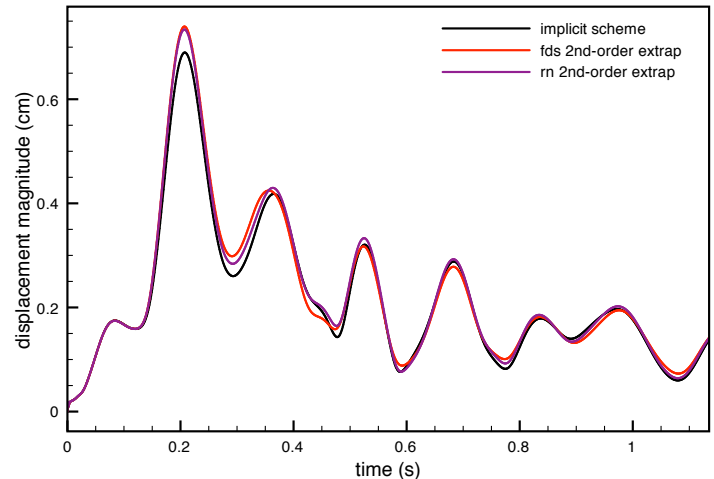

(c)

Figure 17: Maximum displacement magnitude at sections $(a),(b)$ and $(c)$ of the structure vs. time. Comparison of the implicit scheme, Robin-Neumann scheme (from [36, Algorithm 4]) with $r=2$, and Algorithm 2 with $s=0$ and $r=2$.

scheme. The small differences between the explicit Robin-Neumann schemes and Algorithm 2 are due to the different time-seppting of (3) in the fluid: monolithic and projection method $(s=0)$, respectively. Once again, this confirms that the fully decoupled schemes reported in Algorithm 2 preserve the accuracy properties of the original Robin-Neumann schemes introduced in [36]. 


\begin{tabular}{|c|c|c|}
\hline Implicit scheme & Explicit Robin-Neumann schemes ([36]) & Algorithm 2 \\
\hline 10 & 1.2 & 1 \\
\hline
\end{tabular}

Table 4: Elapsed CPU time (dimensionless).

The comparison of the elapsed-CPU times reported in Table 4, and the results of Figures 16 and 17 , plead in favor of the schemes proposed in the present paper with $r=1$ or $r=2$.

\section{Conclusion}

In this work we have introduced a new class of numerical methods for fluid-structure interaction problems involving an incompressible fluid and a general thin-walled viscoelastic structure. The methods proposed allow a fully decoupled time-marching of the complete fluid-solid state: fluid velocity, fluid pressure and solid displacement. The basic ingredients of this new fluid-solid splitting paradigm are:

- projection method in the fluid;

- appropriate fractional-step time-marching of the interface Robin consistency (3), which yields explicit interface Robin conditions for the fluid velocity and pressure sub-steps. The implicit treatment of the solid inertia is enough to guarantee stability;

- fluid stresses are transferred to the thin-walled structure in a standard fashion.

Unconditional stability has been proved for some of the variants $(s=0$ and $r=0,1 ; s=1$ and $r=0$ ) in a representative liner setting involving a general linear viscoelastic shell model (Theorem 1). Though not covered by the stability analysis, numerical evidence has shown that the remaining schemes are stable for a reasonable range of the physical and discretization parameters. The numerical study indicates also that the non-incremental $(s=0)$ or incremental $(s=1)$ nature of the projection scheme in the fluid has a limited impact on the overall accuracy of the methods, which is mainly driven by the consistency of the fluid-solid splitting (determined by the extrapolation order $r=0,1,2)$. The best accuracy and robustness are obtained with $r=1,2$, which retrieve the (optimal) first-order accuracy of the implicit scheme. In particular, the results of Section 4.5 demonstrate, for the first time, that physiological blood flow simulations can be effectively performed with an explicit coupling paradigm.

Further investigation can be oriented in several directions. We can consider the extension to second-order time-marching schemes, the analysis in a fully discrete setting and the derivation of a priori error estimates. Another important problem, not covered by this study, is the case of the coupling with thick-walled solid models or with three-dimensional shell models (see, e.g., $[21,14])$. Note that the latter includes higher-order through-the-thickness effects that overcome, in particular, the common modeling assumptions of Remark 2. An interesting step in this direction could be to combine the ideas recently reported in [35] with the present fully decoupled paradigm.

\section{Acknowledgements}

This work was supported by the French National Research Agency (ANR) through the EXIFSI project (ANR-12-JS01-0004). 


\section{A Example of shell model entering the abstract framework of Section 2.2.1}

We consider the shear-membrane-bending model reported in [21, Section 4.2.2]. In this case, the variational form of $(2)_{2,3}$ is given by the following relation:

$$
\begin{aligned}
\rho^{\mathrm{s}} \epsilon\left(\partial_{t} \dot{\boldsymbol{d}}, \boldsymbol{v}\right)_{\Sigma}+\epsilon\left(\boldsymbol{C}^{\alpha \beta \lambda \xi} \gamma_{\alpha \beta}(\boldsymbol{d}), \gamma_{\lambda \xi}(\boldsymbol{v})\right)_{\Sigma}+ & \frac{\epsilon^{3}}{12}\left(\boldsymbol{C}^{\alpha \beta \lambda \xi} \boldsymbol{\chi}_{\alpha \beta}(\boldsymbol{d}, \boldsymbol{\theta}), \boldsymbol{\chi}_{\lambda \xi}(\boldsymbol{v}, \boldsymbol{\eta})\right)_{\Sigma} \\
& +\epsilon\left(\boldsymbol{D}^{\alpha \lambda} \boldsymbol{\zeta}_{\alpha}(\boldsymbol{d}, \boldsymbol{\theta}), \boldsymbol{\zeta}_{\lambda}(\boldsymbol{v}, \boldsymbol{\eta})\right)_{\Sigma}=-(\boldsymbol{\sigma}(\boldsymbol{u}, p) \boldsymbol{n}, \boldsymbol{v})_{\Sigma}
\end{aligned}
$$

with arbitrary test functions $(\boldsymbol{v}, \boldsymbol{\eta})$ vanishing on $\partial \Sigma$. Here, the convention of summation over repeated indices $\alpha, \beta, \lambda, \xi \in\{1,2\}$ is considered. The quantities $\chi_{\alpha \beta}(\boldsymbol{d}, \boldsymbol{\theta}), \gamma_{\alpha \beta}(\boldsymbol{d})$ and $\boldsymbol{\zeta}_{\alpha}(\boldsymbol{d}, \boldsymbol{\theta})$ represent the covariant components of the bending, membrane and shear strain tensors of the shell, respectively (see, e.g., [21, Section 4.2.2] for further details), and

$$
\boldsymbol{C}^{\alpha \beta \lambda \xi} \stackrel{\text { def }}{=} \frac{E}{2(1+\nu)}\left(\boldsymbol{a}^{\alpha \lambda} \boldsymbol{a}^{\beta \xi}+\boldsymbol{a}^{\alpha \xi} \boldsymbol{a}^{\beta \lambda}+\frac{2 \nu}{1-\nu} \boldsymbol{a}^{\alpha \beta} \boldsymbol{a}^{\lambda \xi}\right), \quad \boldsymbol{D}^{\alpha \lambda} \stackrel{\text { def }}{=} \frac{2 E}{1+\nu} \boldsymbol{a}^{\alpha \lambda},
$$

where $\boldsymbol{a}^{\alpha \beta}$ are the contravariant components of the mid-surface metric tensor, and $E, \nu$ the Young modulus and Poisson ratio of the shell, respectively. In this framework, the abstract operators introduced in (14) are given by:

$$
\begin{aligned}
\left(\boldsymbol{A}_{\boldsymbol{d}}^{\mathrm{e}} \boldsymbol{d}, \boldsymbol{v}\right)_{\Sigma}= & \epsilon\left(\boldsymbol{C}^{\alpha \beta \lambda \xi} \boldsymbol{\gamma}_{\alpha \beta}(\boldsymbol{d}), \boldsymbol{\gamma}_{\lambda \xi}(\boldsymbol{v})\right)_{\Sigma}+\frac{\epsilon^{3}}{12}\left(\boldsymbol{C}^{\alpha \beta \lambda \xi} \boldsymbol{\chi}_{\alpha \beta}(\boldsymbol{d}, \mathbf{0}), \boldsymbol{\chi}_{\lambda \xi}(\boldsymbol{v}, \mathbf{0})\right)_{\Sigma} \\
& +\epsilon\left(\boldsymbol{D}^{\alpha \lambda} \boldsymbol{\zeta}_{\alpha}(\boldsymbol{d}, \mathbf{0}), \boldsymbol{\zeta}_{\lambda}(\boldsymbol{v}, \mathbf{0})\right)_{\Sigma} \\
\left(\boldsymbol{B}_{\boldsymbol{d}}^{\mathrm{e}} \boldsymbol{\theta}, \boldsymbol{v}\right)_{\Sigma}= & \frac{\epsilon^{3}}{12}\left(\boldsymbol{C}^{\alpha \beta \lambda \xi} \boldsymbol{\chi}_{\alpha \beta}(\mathbf{0}, \boldsymbol{\theta}), \boldsymbol{\chi}_{\lambda \xi}(\boldsymbol{v}, \mathbf{0})\right)_{\Sigma}+\epsilon\left(\boldsymbol{D}^{\alpha \lambda} \boldsymbol{\zeta}_{\alpha}(\mathbf{0}, \boldsymbol{\theta}), \boldsymbol{\zeta}_{\lambda}(\boldsymbol{v}, \mathbf{0})\right)_{\Sigma}, \\
\left(\boldsymbol{B}_{\boldsymbol{\theta}}^{\mathrm{e}} \boldsymbol{d}, \boldsymbol{\eta}\right)_{\Sigma}= & \frac{\epsilon^{3}}{12}\left(\boldsymbol{C}^{\alpha \beta \lambda \xi} \boldsymbol{\chi}_{\alpha \beta}(\boldsymbol{d}, \mathbf{0}), \boldsymbol{\chi}_{\lambda \xi}(\mathbf{0}, \boldsymbol{\eta})\right)_{\Sigma}+\epsilon\left(\boldsymbol{D}^{\alpha \lambda} \boldsymbol{\zeta}_{\alpha}(\boldsymbol{d}, \mathbf{0}), \boldsymbol{\zeta}_{\lambda}(\mathbf{0}, \boldsymbol{\eta})\right)_{\Sigma} \\
\left(\boldsymbol{A}_{\boldsymbol{\theta}}^{\mathrm{e}} \boldsymbol{\theta}, \boldsymbol{\eta}\right)_{\Sigma}= & \frac{\epsilon^{3}}{12}\left(\boldsymbol{C}^{\alpha \beta \lambda \xi} \boldsymbol{\chi}_{\alpha \beta}(\mathbf{0}, \boldsymbol{\theta}), \boldsymbol{\chi}_{\lambda \xi}(\mathbf{0}, \boldsymbol{\eta})\right)_{\Sigma}+\epsilon\left(\boldsymbol{D}^{\alpha \lambda} \boldsymbol{\zeta}_{\alpha}(\mathbf{0}, \boldsymbol{\theta}), \boldsymbol{\zeta}_{\lambda}(\mathbf{0}, \boldsymbol{\eta})\right)_{\Sigma} .
\end{aligned}
$$

We do retrieve, in particular, that $\boldsymbol{A}_{\boldsymbol{d}}^{\mathrm{e}}, \boldsymbol{A}_{\boldsymbol{\theta}}^{\mathrm{e}}$ are symmetric and that $\boldsymbol{B}_{\boldsymbol{d}}^{\mathrm{e}}$ is the adjoint of $\boldsymbol{B}_{\boldsymbol{\theta}}^{\mathrm{e}}$ (and viceversa).

The viscous operators $\boldsymbol{A}_{\boldsymbol{d}}^{\mathrm{v}}, \boldsymbol{A}_{\boldsymbol{\theta}}^{\mathrm{v}}, \boldsymbol{B}_{\boldsymbol{d}}^{\mathrm{v}}, \boldsymbol{B}_{\boldsymbol{\theta}}^{\mathrm{v}}$ introduced in (14) depend on the modeling assumptions made on the shell physical dissipation. In the case of a simple Rayleigh modeling of the damping, namely,

$$
\boldsymbol{L}_{\boldsymbol{d}}^{\mathrm{v}}(\dot{\boldsymbol{d}}, \dot{\boldsymbol{\theta}})=\alpha_{0} \rho^{\mathrm{s}} \epsilon \dot{\boldsymbol{d}}+\alpha_{1} \boldsymbol{L}_{\boldsymbol{d}}^{\mathrm{e}}(\dot{\boldsymbol{d}}, \dot{\boldsymbol{\theta}}), \quad \boldsymbol{L}_{\boldsymbol{\theta}}^{\mathrm{v}}(\dot{\boldsymbol{d}}, \dot{\boldsymbol{\theta}})=\alpha_{1} \boldsymbol{L}_{\boldsymbol{\theta}}^{\mathrm{e}}(\dot{\boldsymbol{d}}, \dot{\boldsymbol{\theta}}),
$$

with $\alpha_{0}, \alpha_{1}>0$, the expression of the operators $\boldsymbol{A}_{\boldsymbol{d}}^{\mathrm{v}}, \boldsymbol{A}_{\boldsymbol{\theta}}^{\mathrm{v}}, \boldsymbol{B}_{\boldsymbol{d}}^{\mathrm{v}}, \boldsymbol{B}_{\boldsymbol{\theta}}^{\mathrm{v}}$ follows straightforwardly from the relations (14), (33) and (34).

\section{References}

[1] STACOM2013: 4th International Conference on Statistical Atlases and Computational Models of the Heart. 2013.

[2] M. Astorino, F. Chouly, and M. A. Fernández. Robin based semi-implicit coupling in fluidstructure interaction: Stability analysis and numerics. SIAM J. Sci. Comput., 31(6):40414065, 2009. 
[3] M. Astorino and C. Grandmont. Convergence analysis of a projection semi-implicit coupling scheme for fluid-structure interaction problems. Numer. Math., 116:721-767, 2010.

[4] S. Badia, F. Nobile, and C. Vergara. Fluid-structure partitioned procedures based on Robin transmission conditions. J. Comp. Phys., 227:7027-7051, 2008.

[5] S. Badia, A. Quaini, and A. Quarteroni. Modular vs. non-modular preconditioners for fluid-structure systems with large added-mass effect. Comput. Methods Appl. Mech. Engrg., 197(49-50):4216-4232, 2008.

[6] S. Badia, A. Quaini, and A. Quarteroni. Splitting methods based on algebraic factorization for fluid-structure interaction. SIAM J. Sci. Comput., 30(4):1778-1805, 2008.

[7] H. Baek and G.E. Karniadakis. Sub-iteration leads to accuracy and stability enhancements of semi-implicit schemes for the Navier-Stokes equations. J. Comput. Phys., 230(12):43844402, 2011.

[8] H. Baek and G.E. Karniadakis. A convergence study of a new partitioned fluid-structure interaction algorithm based on fictitious mass and damping. J. Comput. Phys., 231(2):629 $652,2012$.

[9] Satish Balay, Shrirang Abhyankar, Mark F. Adams, Jed Brown, Peter Brune, Kris Buschelman, Victor Eijkhout, William D. Gropp, Dinesh Kaushik, Matthew G. Knepley, Lois Curfman McInnes, Karl Rupp, Barry F. Smith, and Hong Zhang. PETSc Web page, 2014.

[10] Satish Balay, Shrirang Abhyankar, Mark F. Adams, Jed Brown, Peter Brune, Kris Buschelman, Victor Eijkhout, William D. Gropp, Dinesh Kaushik, Matthew G. Knepley, Lois Curfman McInnes, Karl Rupp, Barry F. Smith, and Hong Zhang. PETSc users manual. Technical Report ANL-95/11 - Revision 3.5, Argonne National Laboratory, 2014.

[11] Satish Balay, William D. Gropp, Lois Curfman McInnes, and Barry F. Smith. Efficient management of parallelism in object oriented numerical software libraries. In E. Arge, A. M. Bruaset, and H. P. Langtangen, editors, Modern Software Tools in Scientific Computing, pages 163-202. Birkhäuser Press, 1997.

[12] J.W. Banks, W.D. Henshaw, and D.W. Schwendeman. An analysis of a new stable partitioned algorithm for FSI problems. Part II: Incompressible flow and structural shells. $J$. Comput. Phys., 268:399-416, 2014.

[13] M. Bischoff, K.-U. Bletzinger, W.A. Wall, and E. Ramm. Models and Finite Elements for Thin-Walled Structures, chapter 3. John Wiley \& Sons, Ltd, 2004.

[14] M. Bischoff and E. Ramm. On the physical significance of higher order kinematic and static variables in a three-dimensional shell formulation. Int. J. Solids Structures, 37:6933-6960, 2000 .

[15] F. Brezzi and J. Pitkäranta. On the stabilization of finite element approximations of the Stokes equations. In Efficient solutions of elliptic systems (Kiel, 1984), volume 10 of Notes Numer. Fluid Mech., pages 11-19. Vieweg, 1984.

[16] D.L. Brown, R. Cortez, and M.L. Minion. Accurate projection methods for the incompressible Navier-Stokes equations. J. Comput. Phys., 168(2):464-499, 2001. 
[17] M. Bukač, S. Čanić, R. Glowinski, J. Tambača, and A. Quaini. Fluid-structure interaction in blood flow capturing non-zero longitudinal structure displacement. J. Comput. Phys., 235:515-541, 2013.

[18] E. Burman and M.A. Fernández. Stabilization of explicit coupling in fluid-structure interaction involving fluid incompressibility. Comput. Methods Appl. Mech. Engrg., 198(5-8):766784, 2009 .

[19] E. Burman and M.A. Fernández. Explicit strategies for incompressible fluid-structure interaction problems: Nitsche type mortaring versus Robin-Robin coupling. Int. J. Num. Meth. Engrg., 2013. In press.

[20] P. Causin, J.-F. Gerbeau, and F. Nobile. Added-mass effect in the design of partitioned algorithms for fluid-structure problems. Comput. Methods Appl. Mech. Engrg., 194(4244):4506-4527, 2005.

[21] D. Chapelle and K.J. Bathe. The Finite Element Analysis of Shells - Fundamentals. Springer, 2011.

[22] D. Chapelle and A. Ferent. Modeling of the inclusion of a reinforcing sheet within a 3D medium. Math. Models Methods Appl. Sci., 13(4):573-595, 2003.

[23] A.J. Chorin. Numerical solution of the Navier-Stokes equations. Math. Comp., 22:745-762, 1968.

[24] P. Crosetto, S. Deparis, G. Fourestey, and A. Quarteroni. Parallel algorithms for fluidstructure interaction problems in haemodynamics. SIAM J. Sci. Comput., 33(4):1598-1622, 2011.

[25] J. Degroote. Partitioned simulation of fluid-structure interaction. Arch. Comput. Method E., 20(3):185-238, 2013.

[26] P. Degroote, J. Bruggeman, R. Haelterman, and J. Vierendeels. Stability of a coupling technique for partitioned solvers in FSI applications. Comp. 83 Struct., 86(23-24):2224$2234,2008$.

[27] J. Donéa, S. Giuliani, and J. P. Halleux. An arbitrary Lagrangian-Eulerian finite element method for transient dynamic fluid-structure interactions. Comp. Meth. Appl. Mech. Engng., pages 689-723, 1982.

[28] M. Eswaran, U.K. Saha, and D. Maity. Effect of baffles on a partially filled cubic tank: Numerical simulation and experimental validation. Computers $\& 3$ Structures, 87(3-4):198$205,2009$.

[29] M.A. Fernández. Coupling schemes for incompressible fluid-structure interaction: implicit, semi-implicit and explicit. S $\overrightarrow{\mathrm{e}} M A$ J., 55(1):59-108, 2011.

[30] M.A. Fernández. Incremental displacement-correction schemes for the explicit coupling of a thin structure with an incompressible fluid. C. R. Math. Acad. Sci. Paris, 349(7-8):473-477, 2011.

[31] M.A. Fernández. Incremental displacement-correction schemes for incompressible fluidstructure interaction: stability and convergence analysis. Numer. Math., 123(1):21-65, 2013. 
[32] M.A. Fernández, J.F. Gerbeau, and C. Grandmont. A projection semi-implicit scheme for the coupling of an elastic structure with an incompressible fluid. Int. J. Num. Meth. Engrg., 69(4):794-821, 2007.

[33] M.A. Fernández and M. Landajuela. A fully decoupled scheme for the interaction of a thin-walled structure with an incompressible fluid. C. R. Math. Acad. Sci. Paris, 351(34):161-164, 2013

[34] M.A. Fernández and M. Moubachir. A Newton method using exact Jacobians for solving fluid-structure coupling. Comp. \& Struct., 83:127-142, 2005.

[35] M.A. Fernández, J. Mullaert, and M. Vidrascu. Generalized Robin-Neumann explicit coupling schemes for incompressible fluid-structure interaction: stability analysis and numerics. Internat. J. Numer. Methods Engrg. (2014) doi: 10.1002/nme.4785.

[36] M.A. Fernández, J. Mullaert, and M. Vidrascu. Explicit Robin-Neumann schemes for the coupling of incompressible fluids with thin-walled structures. Comput. Methods Appl. Mech. Engrg., 267:566-593, 2013.

[37] L. Formaggia, A. Quarteroni, and A. Veneziani, editors. Cardiovascular Mathematics. Modeling and simulation of the circulatory system, volume 1 of Modeling, Simulation and Applications. Springer, 2009.

[38] M.W. Gee, Ch. Förster, and W.A. Wall. A computational strategy for prestressing patientspecific biomechanical problems under finite deformation. Int. J. Numer. Meth. Biomed. Engng., 26(1):52-72, 2010.

[39] M.W. Gee, U. Küttler, and W. Wall. Truly monolithic algebraic multigrid for fluid-structure interaction. Int. J. Numer. Meth. Engng., 85(8):987-1016, 2011.

[40] J.-L. Guermond. Some implementation of projection methods for Navier-Stokes equations. M2AN Math. Model. Numer. Anal., 30:637-667, 1996.

[41] J. L. Guermond, P. Minev, and J. Shen. An overview of projection methods for incompressible flows. Comput. Methods Appl. Mech. Engrg., 195(44-47):6011-6045, 2006.

[42] G. Guidoboni, R. Glowinski, N. Cavallini, and S. Čanić. Stable loosely-coupled-type algorithm for fluid-structure interaction in blood flow. J. Comp. Phys., 228(18):6916-6937, 2009 .

[43] F. Hecht. New development in FreeFem++. J. Numer. Math., 20(3-4):251-265, 2012.

[44] M. Heil and A.L. Hazel. Fluid-structure interaction in internal physiological flows. In Annual review of fluid mechanics. Volume 43, 2011, volume 43 of Annu. Rev. Fluid Mech., pages 141-162. Annual Reviews, 2011.

[45] G.A. Holzapfel. Nonlinear solid mechanics. John Wiley \& Sons, Ltd., Chichester, 2000.

[46] G. Hou, J. Wang, and A. Layton. Numerical methods for fluid-structure interaction-a review. Commun. Comput. Phys., 12(2):337-377, 2012.

[47] http://felisce.gforge.inria.fr.

[48] U. Küttler, C. Förster, and W.A. Wall. A solution for the incompressibility dilemma in partitioned fluidâĂŞstructure interaction with pure Dirichlet fluid domains. Comput. Mech., 38:417-429, 2006. 
[49] P. Le Tallec. Domain decomposition methods in computational mechanics. In J. Tinsley Oden, editor, Computational Mechanics Advances, volume 1 (2), pages 121-220. NorthHolland, 1994.

[50] P. Le Tallec and M. Vidrascu. Solving large scale structural problems on parallel computers using domain decomposition techniques, chapter 3, pages 49-82. J. Wiley, 1996.

[51] J. Liu, R.K. Jaiman, and P.S. Gurugubelli. A stable second-order scheme for fluid-structure interaction with strong added-mass effects. J. Comput. Phys., 270:687-710, 2014.

[52] M. Lombardi, N. Parolini, A. Quarteroni, and G. Rozza. Numerical simulation of sailing boats: Dynamics, FSI, and shape optimization. In G. Buttazzo and A. Frediani, editors, Variational Analysis and Aerospace Engineering: Mathematical Challenges for Aerospace Design, Springer Optimization and Its Applications, pages 339-377. Springer, 2012.

[53] M. Lukáčová-Medvid'ová, G. Rusnáková, and A. Hundertmark-Zaušková. Kinematic splitting algorithm for fluid-structure interaction in hemodynamics. Comput. Methods Appl. Mech. Engrg., 265(1):83-106, 2013.

[54] J. Mandel. Balancing domain decomposition. Comm. Numer. Methods Engrg., 9(3):233-241, 1993.

[55] P. Moireau, N. Xiao, M. Astorino, C.A. Figueroa, D. Chapelle, C.A. Taylor, and J-F. Gerbeau. External tissue support and fluid-structure simulation in blood flows. Biomech. Model. Mechanobiol., 11:1-18, 2012.

[56] R.L. Muddle, M. Mihajlović, and M. Heil. An efficient preconditioner for monolithicallycoupled large-displacement fluid-structure interaction problems with pseudo-solid mesh updates. J. Comput. Phys., 231(21):7315-7334, 2012.

[57] F. Nobile, M. Pozzoli, and C. Vergara. Time accurate partitioned algorithms for the solution of fluidâĂŞstructure interaction problems in haemodynamics. Comput. Fluids, 86:470-482, 2013.

[58] F. Nobile, M. Pozzoli, and C. Vergara. Inexact accurate partitioned algorithms for fluidstructure interaction problems with finite elasticity in haemodynamics. J. Comput. Phys., 273:598-617, 2014.

[59] F. Nobile and C. Vergara. An effective fluid-structure interaction formulation for vascular dynamics by generalized Robin conditions. SIAM J. Sci. Comput., 30(2):731-763, 2008.

[60] S. Oyre, E.M. Pedersen, S. Ringgaard, P. Boesiger, and W.P. Paaske. In vivo wall shear stress measured by magnetic resonance velocity mapping in the normal human abdominal aorta. Eur. J. Vasc. Endovasc. Surg., 13:263-271, 1997.

[61] M.P. Païdoussis, S.J. Price, and E. de Langre. Fluid-structure interactions: cross-flowinduced instabilities. Cambridge University Press, 2011.

[62] S. Pant, B. Fabrèges, J.-F. Gerbeau, and I.E. Vignon-Clementel. A methodological paradigm for patient-specific multi-scale CFD simulations: from clinical measurements to parameter estimates for individual analysis. Int. J. Numer. Meth. Biomed. Engng. doi: $10.1002 / \mathrm{cnm} .2692$.

[63] C. Pozrikidis. Computational hydrodynamics of capsules and biological cells. Chapman \& Hall/CRC Mathematical and Computational Biology. CRC Press, 2010. 
[64] A. Prohl. Projection and quasi-compressibility methods for solving the incompressible NavierStokes equations. Advances in Numerical Mathematics. B.G. Teubner, Stuttgart, 1997.

[65] A. Quaini and A. Quarteroni. A semi-implicit approach for fluid-structure interaction based on an algebraic fractional step method. Math. Models Methods Appl. Sci., 17(6):957-983, 2007.

[66] Th. Richter and Th. Wick. Finite elements for fluidâĂŞstructure interaction in ALE and fully eulerian coordinates. Comput. Methods Appl. Mech. Engrg., 199(41âĂŞ44):2633-2642, 2010 .

[67] A.-V. Salsac, S.R. Sparks, J.M. Chomaz, and J.C. Lasheras. Evolution of the wall shear stresses during the progressive enlargement of symmetric abdominal aortic aneurysms. $J$. Fluid Mech., 550:19-51, 2006.

[68] B. Smith, P. Bjorstad, and W. Gropp. Domain Decomposition: Parallel Multilevel Methods for Elliptic Partial Differential Equations. Cambridge University Press, 1996.

[69] K. Takizawa and T.E. Tezduyar. Computational methods for parachute fluid-structure interactions. Arch. Comput. Methods Eng., 19:125-169, 2012.

[70] R. Temam. Une méthode d'approximation de la solution des équations de Navier-Stokes. Bull. Soc. Math. France, 96:115-152, 1968.

[71] J. Young and S. Mitran. A numerical model of cellular blebbing: A volume-conserving, fluid-structure interaction model of the entire cell. J. Biomech., 43(2):210-220, 2010. 


\section{Contents}

1 Introduction $\quad 3$

2 Derivation and analysis in the linear case $\quad 4$

2.1 Time semi-discretization: fully decoupled schemes . . . . . . . . . . . . . 5

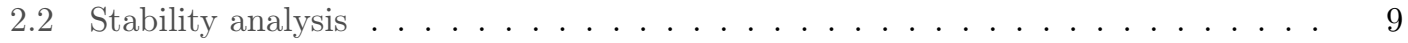

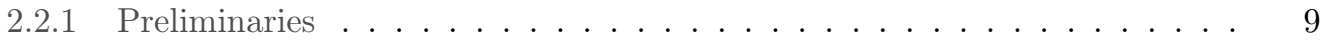

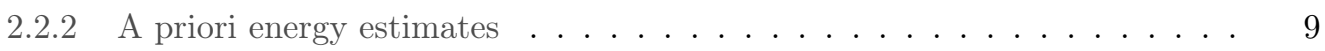

3 The non-linear case $\quad 14$

4 Numerical experiments $\quad 16$

4.1 Convergence study in a two-dimensional test-case . . . . . . . . . . . . . . . . 16

4.2 Pressure wave propagation in a straight tube . . . . . . . . . . . . . . . . . 19

4.3 Damped structural instability with a fully enclosed fluid . . . . . . . . . . . . . 21

4.4 Blood flow in an abdominal aortic aneurysm . . . . . . . . . . . . . . . . . . 23

4.5 Blood flow in a patient-specific aorta . . . . . . . . . . . . . 25

5 Conclusion $\quad 31$

A Example of shell model entering the abstract framework of Section 2.2.1 32 


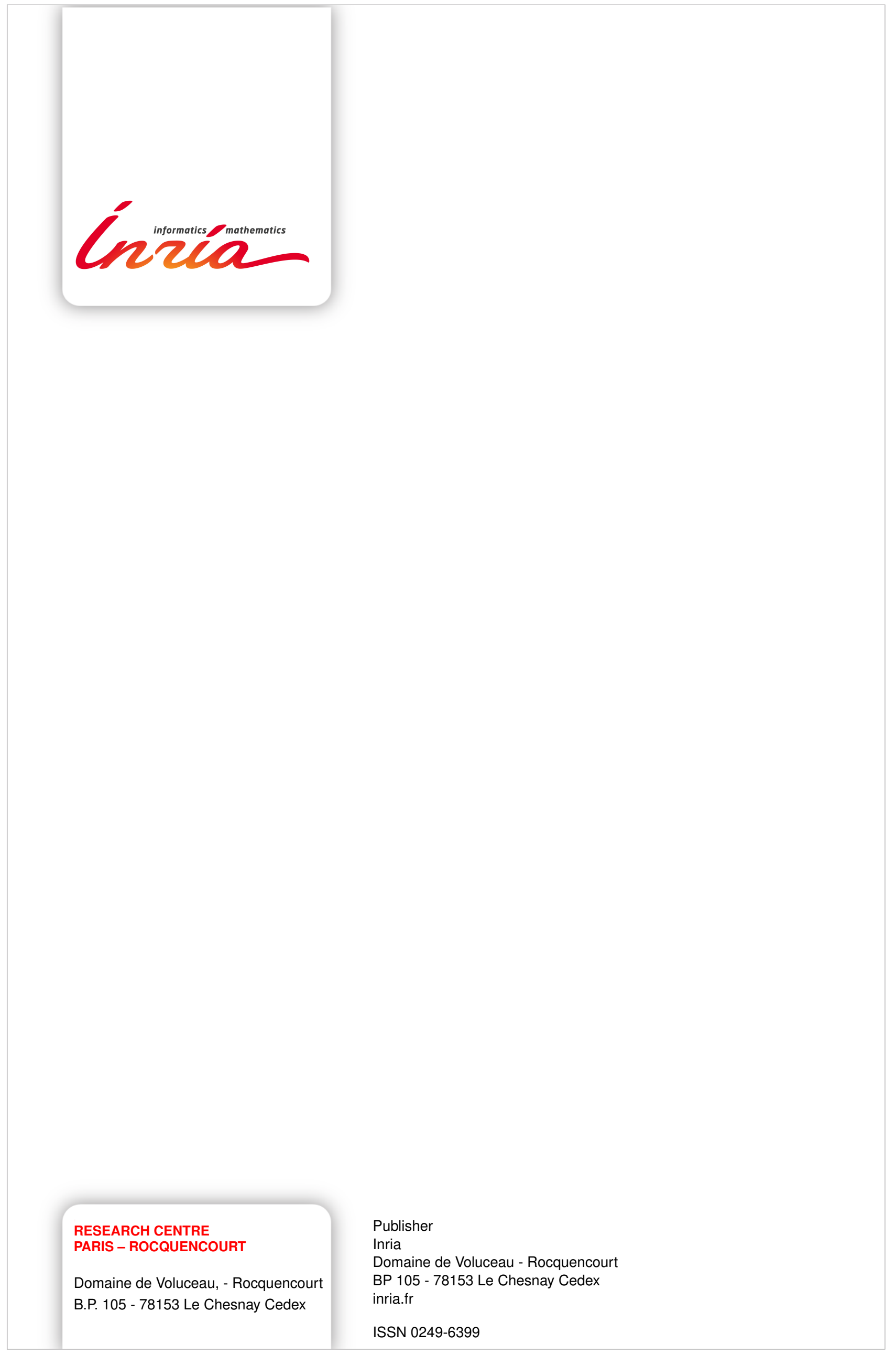

